\title{
Identification of transcription factor binding sites using ATAC-seq
}

\author{
Zhijian Li ${ }^{1,2}$, Marcel H. Schulz 3,4,5,6, Thomas Look ${ }^{2,7}$, Matthias Begemann ${ }^{8}$, Martin Zenke ${ }^{2,7}$ \\ and Ivan G. Costa ${ }^{1,7^{*}}$ (i)
}

\begin{abstract}
Transposase-Accessible Chromatin followed by sequencing (ATAC-seq) is a simple protocol for detection of open chromatin. Computational footprinting, the search for regions with depletion of cleavage events due to transcription factor binding, is poorly understood for ATAC-seq. We propose the first footprinting method considering ATAC-seq protocol artifacts. HINT-ATAC uses a position dependency model to learn the cleavage preferences of the transposase. We observe strand-specific cleavage patterns around transcription factor binding sites, which are determined by local nucleosome architecture. By incorporating all these biases, HINT-ATAC is able to significantly outperform competing methods in the prediction of transcription factor binding sites with footprints.
\end{abstract}

Keywords: Computational footprinting, Open chromatin, ATAC-seq, Cleavage bias

\section{Background}

DNase-I hypersensitive sites sequencing (DNase-seq; [1-4]) and Assays for Transposase-Accessible Chromatin sequencing (ATAC-seq; $[5,6]$ ) are two widely used protocols for genome-wide identification of open chromatin. DNase-seq and ATAC-seq are based on the use of cleavage enzymes (DNase-I and Tn5, respectively), which recognize and cleave DNA in open chromatin regions. Sequencing and the alignment of reads from these fragments allows the detection of open chromatin by identifying genomic intervals with many reads $[1,2]$. However, the presence of transcription factors (TFs) bound to the DNA prevents the enzyme from cleavage in an otherwise nucleosome-free region. This leaves small regions, referred to as footprints, where read coverage suddenly drops within peak regions of high coverage.

Computational methods scanning open chromatin profiles to find footprints have been shown to predict transcription factor binding sites (TFBS) with high accuracy in DNase-seq data [7, 8]. Among others, computational footprinting has been used to detect the regulatory lexicon of

\footnotetext{
*Correspondence: ivan.costa@rwth-aachen.de

${ }^{1}$ Institute for Computational Genomics, Joint Research Center for

Computational Biomedicine, RWTH Aachen University Medical School, 52074

Aachen, Germany

${ }^{7}$ Helmholtz Institute for Biomedical Engineering, RWTH Aachen University,

Aachen, Germany

Full list of author information is available at the end of the article
}

several cell types $[9,10]$, to measure the effects of genetic variants in TF binding [11] and to assess changes in the activity of TFs, e.g., during inflammatory responses [12] or fasting conditions [13]. Computational footprinting, which only requires a single open chromatin experiment per cell of interest, is a powerful tool to study regulatory processes.

ATAC-seq has several experimental advantages over DNase-seq: it requires fewer cells (50.000 to single cells) and is less laborious $[5,6]$. Not surprisingly, the number of ATAC-seq-based studies deposited in Gene Expression Omnibus is twelve times higher than the number of DNase-seq-based studies in the last year (366 ATAC-seq vs. 29 DNase-seq $)^{1}$. There is also two times more ATACseq samples than DNase-seq samples per study, confirming that its experimental simplicity makes it a good choice for studies with large sample size, for example in clinical settings [14]. However, computational footprinting is still poorly explored in ATAC-seq data. The single study contrasting ATAC-seq and DNase-seq shows that ATAC-seq footprints have inferior accuracy than DNaseseq footprints [15]. It was also reported that ATAC-seq average footprint profiles are not so well defined as average footprint profiles from DNase-seq [11]. However, all the work with footprinting in ATAC-seq so far $[5,15,16]$ used computational methods tailored to DNase-seq data

(c) The Author(s). 2019 Open Access This article is distributed under the terms of the Creative Commons Attribution 4.0 International License (http://creativecommons.org/licenses/by/4.0/), which permits unrestricted use, distribution, and reproduction in any medium, provided you give appropriate credit to the original author(s) and the source, provide a link to the Creative Commons license, and indicate if changes were made. The Creative Commons Public Domain Dedication waiver (http://creativecommons.org/publicdomain/zero/1.0/) applies to the data made available in this article, unless otherwise stated. 
and ignored characteristics intrinsic to the ATAC-seq protocol.

A possible reason for the lower performance of ATACseq footprinting might be the cleavage enzyme Tn 5 itself, which has a large (17bp) “Tn5 motif" $[5,17]$ and a complex cleavage mechanism requiring a Tn5 dimer for action. The large size of the Tn5 dimer makes cleavage events dependent on structural features of the neighboring proteins (TFs or histones) and on the size of accessible DNA [18]. Cleavage events in small linker DNA between nucleosomes are possible, but less likely than cleavage of fragments from active regulatory regions [5]. Importantly, the DNA binding preferences of enzymes cause sequence-specific cleavage bias. Thus, computational bias correction is an important aspect of the analysis of DNaseseq $[19,20]$ and ATAC-seq data [21]. Some work uses position weight matrices (PWMs), which assume independence between positions, to model DNase-seq bias [22]. However, most bias correction methods infer bias estimates using $k$-mer sequences around the start of aligned reads, by estimating the probability of finding a $k$ mer at read start sites against occurrences in the genome [19]. For DNase-seq, a $k$ equal to 6 was frequently used $[8,11,19,20,23]$. This method requires the estimation of a multinomial distribution and is likely to suffer from overfitting for large $k$-mers [24]. Alternatively, position dependency models (PDMs) allow flexibility in the type of dependencies being modeled [25, 26]. They have been shown to overcome the problem of overfitting in modeling protein-DNA binding preferences. We are unaware of methods exploring effects of the local chromatin structure in ATAC-seq or the use of PDMs for modeling the bias of cleavage enzymes.

Here, we propose HINT-ATAC, which is the first footprinting method dealing with the characteristics of the ATAC-seq protocol. First, we propose the use of a probabilistic PDM based on sparse local inhomogeneous mixtures (SLIM) models for the correction of cleavage bias [26] and evaluate it for both ATAC-seq and DNase-seq protocols. Second, we model a novel observation that ATAC-seq cleavage events show a strand bias, which is associated to the number of nucleosomes in ATAC-seq fragments. HINT-ATAC, which is based on hidden Markov models, uses strand-specific, nucleosome-size decomposed, and bias-corrected signals to identify footprints. We show that HINT-ATAC significantly improves the recovery of footprints supported by TF ChIP-seq data [8, 27] from ENCODE cell lines [9]. Moreover, HINT-ATAC footprints have similar predictive accuracy using either ATAC-seq or DNase-seq protocols. Finally, as an example of practical application of footprint analysis, we use HINT-ATAC to detect TFs associated with immune dendritic cell (DC) specification.

\section{Results}

\section{The transposase Tn 5 has a complex cleavage bias}

Cleavage bias is caused by the preference of enzymes to cleave particular DNA sequences [19] as indicated by the motifs around the start sites of DNase-seq and ATAC-seq reads (Fig. 1a, b). Motifs are similar for distinct ATAC-seq libraries and protocol variations such as standard [5], Omni-[6], and Fast-ATAC [28] (Additional file 1: Figure S1). The Tn5 dimer cleaves the DNA by inserting two distinct adapters in the DNA fragment ends. Cleavage leaves two 9 bps single-strand DNA ends that are later extended in the ATAC-seq protocol (Fig. 1c). The fact that Tn 5 works as a dimer, where two Tn 5 proteins bind to the DNA in reverted orientations, causes the large (9-13 bps) palindromic Tn5 motif (Fig. 1a). Moreover, the motif is centered around position +5 relative to the read start, which represents the middle position of the Tn 5 cleavage event. In contrast, DNase-I leaves a short motif close to the start of reads in DNase-seq experiments (Fig. 1b).

\section{Position dependency models improve cleavage bias correction}

We evaluate here the use of position dependency models (PDM) to estimate the bias of Tn5 cleavage events. HINTATAC considers the fifth base of ATAC-seq reads as the cleavage event as in [5]. A PDM learns relevant dependencies from the data and is less likely to overfit than $k$-mer-based approaches, when large sequences need to be considered. We compare the performance of PDMs to $k$ mer or PWM-based bias correction, the two methods previously used in the literature (see the "Method" section). This includes an analysis of the optimal word size $k$ necessary to capture cleavage bias for both Tn 5 and DNase-I. Cleavage signals obtained by distinct correction methods (and uncorrected signals) are given as input for the footprinting method HINT [7] (Additional file 1: Figure S2). We then evaluate the recovery of footprints with binding sites supported by ChIP-seq peaks on 32 TFs from the GM12878 cell (training dataset). For this, we calculate the area under precision recall curve (AUPR) and the area under receiver operating characteristics curve (AUC) for distinct false positive rates (1\%, 10\%, and 100\%) for each $\mathrm{TF}$ as in [8]. A final ranking score is obtained by combining the ranking of a method for each of the six statistics. A higher ranking score indicates higher recovery of ChIP-seq supported footprints.

The comparative analysis indicates that PDMs are best for footprint detection in all evaluated libraries with the exception of Omni ATAC-seq, where $k$-mer and PDM tied first (Fig. 2a, b, Additional file 1: Figure S4). One important question is the robustness of methods when estimated on libraries with different sequencing depths. Therefore, we perform random under-sampling of an ATAC-seq library by decreasing its size from 


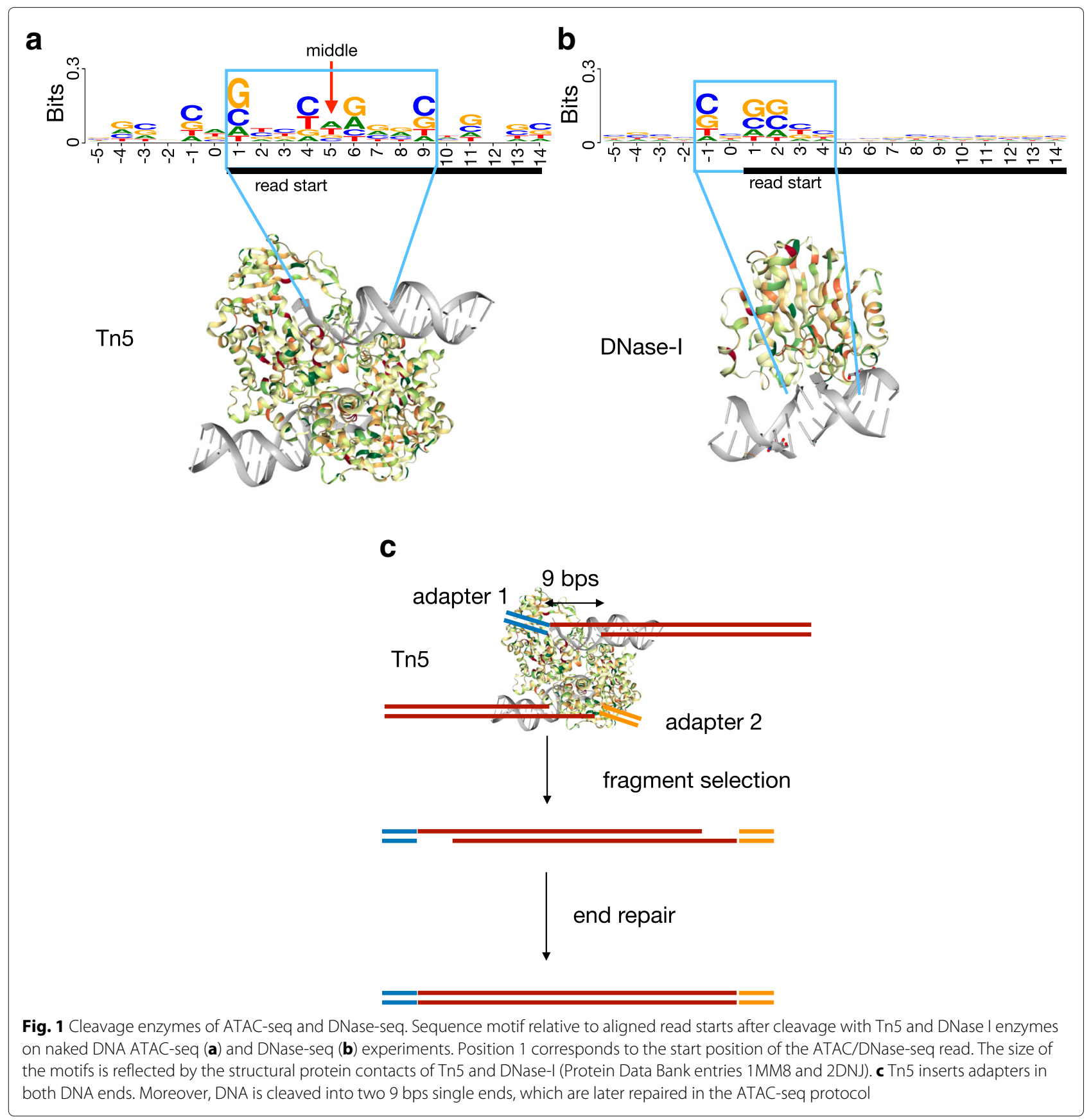

70 to 35 million reads. We observe that the PDM is ranked first when considering only $75 \%$ or $50 \%$ of Omni-ATAC-seq reads (Additional file 1: Figure S4). Moreover, bias estimates from PDMs remain highly similar after under-sampling, while $k$-mer estimates show increasing variance with less reads (Additional file 1: Figure S5). Another relevant question is the size of the sequence $(k)$, which needs to be considered for capturing cleavage bias. Interestingly, smaller sequences (4-8) are selected for DNase-seq data, while larger sequences (8-12) are best for ATAC-seq protocols (Fig. 2a, b).

These results fit with the observation that the most conserved positions in the Tn5 motif, i.e., positions 1 and 9 in Fig. 1a, are farther away than in the DNase-I motif, i.e., positions -1 and 2 . As a consequence, a larger sequence size is necessary to correct the Tn5 bias. We also observe that the distribution of cleavage bias estimates is more dispersed in ATAC-seq than in DNase-seq (Additional file 1: Figure S6), which indicates more 

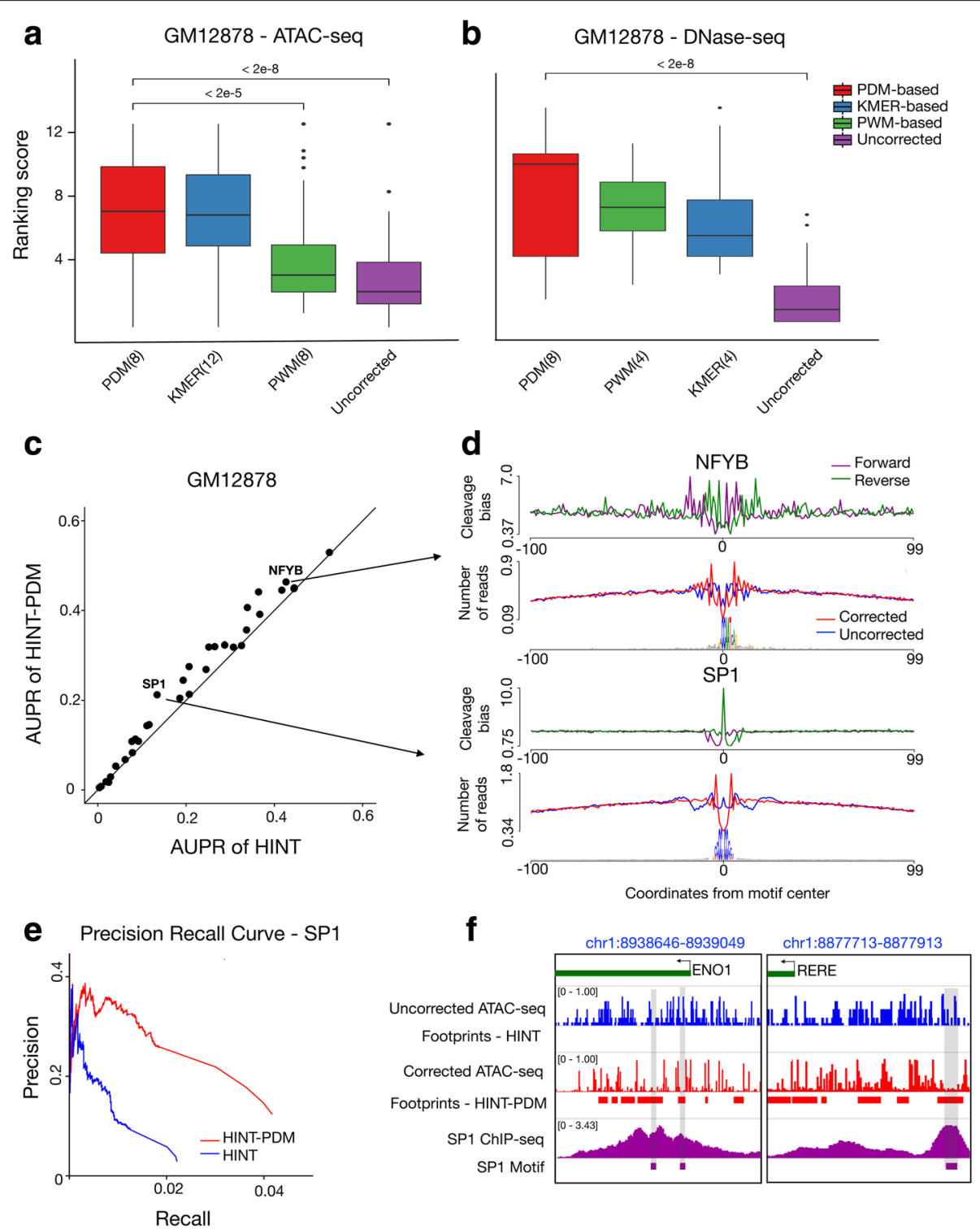

Fig. 2 Strategies for cleavage bias correction. Comparison of bias estimation methods in standard ATAC-seq (a) and DNase-seq (b) on 32 TF ChIP-seq data sets from GM12878 cells. The $y$-axis denotes the ranking score, where higher values indicate higher recovery of footprints supported by TF ChIP-seq peaks. Numbers after methods names ( $x$-axis) indicate optimal word size ( $k$ ). $p$ values are based on the Friedman-Nemenyi test (see Additional file 1: Table S1-S12 for complete results). c The scatter plot contrasting AUPR of HINT with PDM-based estimation with 8-mers ( $y$-axis) and HINT without bias correction ( $x$-axis) in GM12878 cells. d Bias estimates and average ATAC-seq signals centered around NFYB and SP1 motifs supported by a ChIP-seq peaks in GM12878 cells. e Precision-recall curve also supports the improvement in prediction of SP1 ChIP-Seq supported binding sites with cleavage bias correction. f ATAC-seq cleavage signals and footprint predictions with (HINT-PDM) and without (HINT) bias correction in two selected genomic regions. Footprint predictions on bias-corrected signals match SP1 motifs supported by ChIP-seq peaks, while no footprints are predicted in uncorrected ATAC-seq due to the presence of cleavage sites within the SP1 motif

extreme bias for particular sequences for ATAC-seq libraries. Another important question is the dependencies between sequence positions, which are observed on parameters learned by PDMs or by a statistical test proposed in [29]. While neighboring (first order) dependencies are most relevant for both Tn 5 and DNase-I enzymes, a few higher order dependencies are relevant for ATACseq (Additional file 1: Figure S7). These results support the fact that PDMs, which learn relevant dependencies from the data, are more suitable for modeling the bias of Tn5. We adopt the use of PDM with $k=8$ as standard in HINT-ATAC in all experiments below. 
Another relevant question is the use of ATAC-seq and DNase-seq libraries performed on naked DNA, which were previously proposed as control libraries for measurement of cleavage bias [30]. However, results indicate that bias estimates based on GM12878 cells have higher ranks than the use of naked DNA (Additional file 1: Figure S8). This is also supported by the similarity of bias estimates on several ATAC-seq libraries, where bias estimates based on the naked DNA group apart from bias obtained in the libraries themselves (Additional file 1: Figure S8). Given that cleavage bias varies in distinct degrees for each library, these results support the use of bias estimates based on reads from the ATAC-seq library at hand.

\section{Cleavage bias estimates and TF-specific impact}

It was previously shown that the impact of cleavage bias correction is TF-specific $[8,19,30]$, i.e., TFs with motifs similar to the enzyme motif will not leave clear footprints. Therefore, we compare the AUPR of 32 individual TFs predicted with bias-corrected and uncorrected signals (Fig. 2c). Most of the TFs (29 out of 32) have an increase in AUPR, while the AUPR decrease for 3 TFs is marginal (average of 0.003; Additional file 1: Figure S9). As expected, TFs with the highest increase in AUPR (NFYB and Sp1 Fig. 2d, e) have depletion of ATAC-seq cleavage sites around their binding sites after bias correction. Moreover, ATAC-seq profiles in individual genomic locations also support the advantage of cleavage bias correction in the detection of footprints (Fig. 2f).

\section{Incorporation of nucleosome density and strand information improves footprinting}

We observe strong strand-specific patterns on average ATAC-seq profiles around CTCF ChIP-seq peaks (Fig. 3a), which was not reported before for ATAC-seq data. This is also observed in individual genomic loci with CTCF-binding sites (Additional file 1: Figure S10). As strand specificity is particularly high in linker regions, we reason that it could be associated with the number of nucleosomes included in the ATAC-seq fragment. Sizes of ATAC-seq fragments, which can be estimated from paired-end sequencing libraries, have typical modal distributions associated to fragments with zero, one, two, or more nucleosomes [5]. We observe that distinct ATACseq protocols have slightly distinct fragment size distributions, which reflect their bias towards more (or less) nucleosome-containing fragments (Fig. 3b).

Therefore, we decompose ATAC-seq cleavage signals by only considering reads from nucleosome-free fragments or reads from fragments with a particular number of nucleosomes (Additional file 1: Figure S11). We evaluate the performance of HINT-ATAC by providing ATAC-seq signals with reads from distinct decomposition strategies (fragment sizes) as input and by varying the number of
HMM states (see the "Method" section and Additional file 1: Figure S12). This includes considering all reads (all), nucleosome-free fragments ( $\mathrm{Nfr}$ ), nucleosome-free and nucleosome-containing fragments, $(\mathrm{Nfr} \&+1 \mathrm{~N})$ and nucleosome-free, one nucleosome, and two or more nucleosomes fragments ( $\mathrm{Nfr} \& 1 \mathrm{~N} \&+2 \mathrm{~N})$. We further evaluate the use of strand-specific and non-strand specific signals, where the dimensions of input signals vary from 2 to $12^{2}$. HINT-ATAC models are evaluated on the prediction of 32 TFs in GM12878 cells (training dataset).

The comparative evaluation indicates that using Nfr is best for standard ATAC and Fast-ATAC protocols, while the combined use of nucleosome-free and nucleosomecontaining signals $(\mathrm{Nfr} \&+1 \mathrm{~N})$ is best for Omni-ATAC (Fig. 3c, Additional file 1: Figure S13). Moreover, optimal HMM models are always based on strand-specific signals. These results indicate the importance of considering fragment sizes and strand information for improving footprint detection in ATAC-seq data. From here on, we will use the optimal HMM configuration for each ATAC-seq protocol determined on the training dataset.

An inspection of the parameters of the Omni-ATAC HMM gives insights on how HINT-ATAC uses nucleosome decomposed cleavage signals to detect footprints (Additional file 1: Figure S14). This HMM includes states associated to the footprint, flanking regions left/right of the footprint and background regions. Interestingly, the left flanking state has high emission values for $\mathrm{Nfr}$ forward reads and $+1 \mathrm{~N}$ reverse reads, while the right flanking state has high emission values on Nfr reverse reads and $+1 \mathrm{~N}$ forward reads. This indicates the importance of strand-specific signals and the presence of reverted strand-specific cleavage patterns on reads from nucleosome-free and nucleosome-containing fragments around the footprint.

\section{Local nucleosome architecture and strand-specific ATAC-seq cleavage profiles}

Previous results indicate that the combination of strandspecific signals and decomposition by nucleosome numbers significantly improves footprint prediction in all ATAC-seq protocols. To understand the mechanism behind strand bias, we define types of ATAC-seq cleavage events relative to the location of the TF binding site (Fig. 4a). Next, we measure the amount of the strand cleavage bias for distinct fragment sizes ( $\mathrm{Al}$, Nfr, $1 \mathrm{~N}$ and $+2 \mathrm{~N}$ ) around distinct intervals near the TF binding site (Additional file 1: Figure S15). We observe in Fig. 4b that there are more forward reads left to CTCF binding in nucleosome-free fragments (forward/reverse ratio of 2.6), while there are more reverse reads left of CTCF for nucleosome-containing fragments (ratios of 0.63 for $1 \mathrm{~N}$ and 0.5 for $+2 \mathrm{~N}$ ). This bias is not so prominent when 


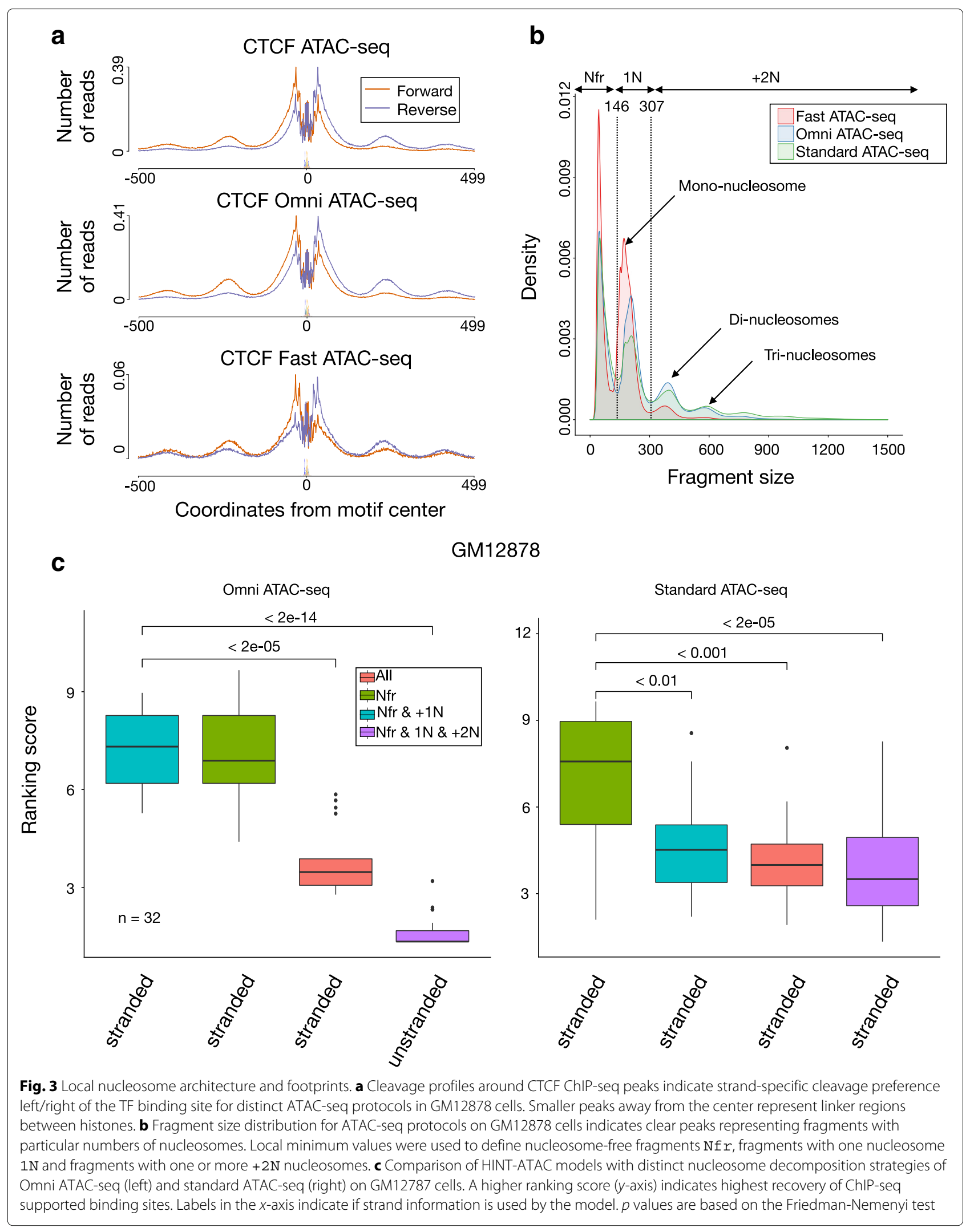


a
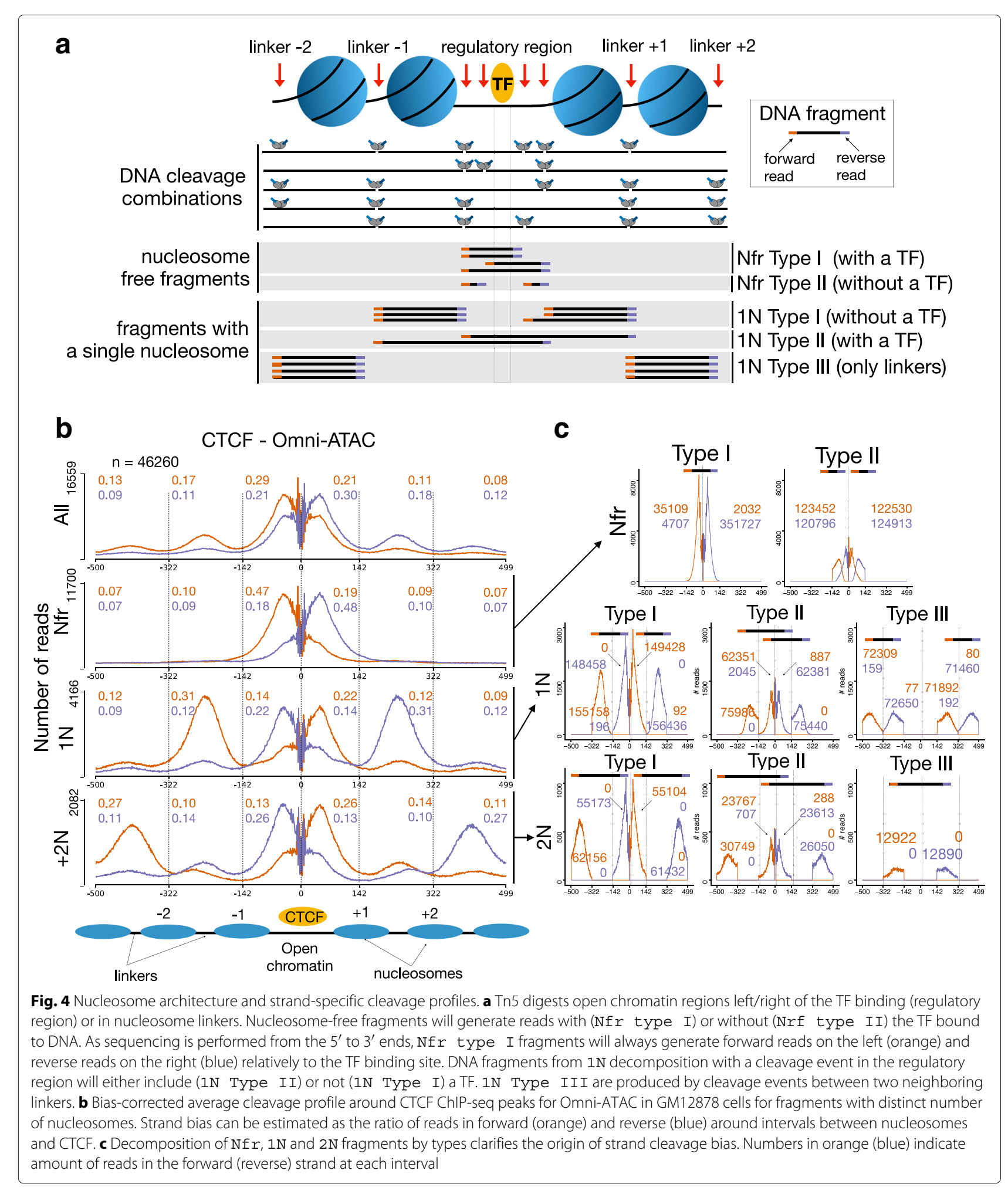

b DNA cleavage
combinations

DNA fragment forward reverse nucleosome free fragments INfr Type II (without a TF)

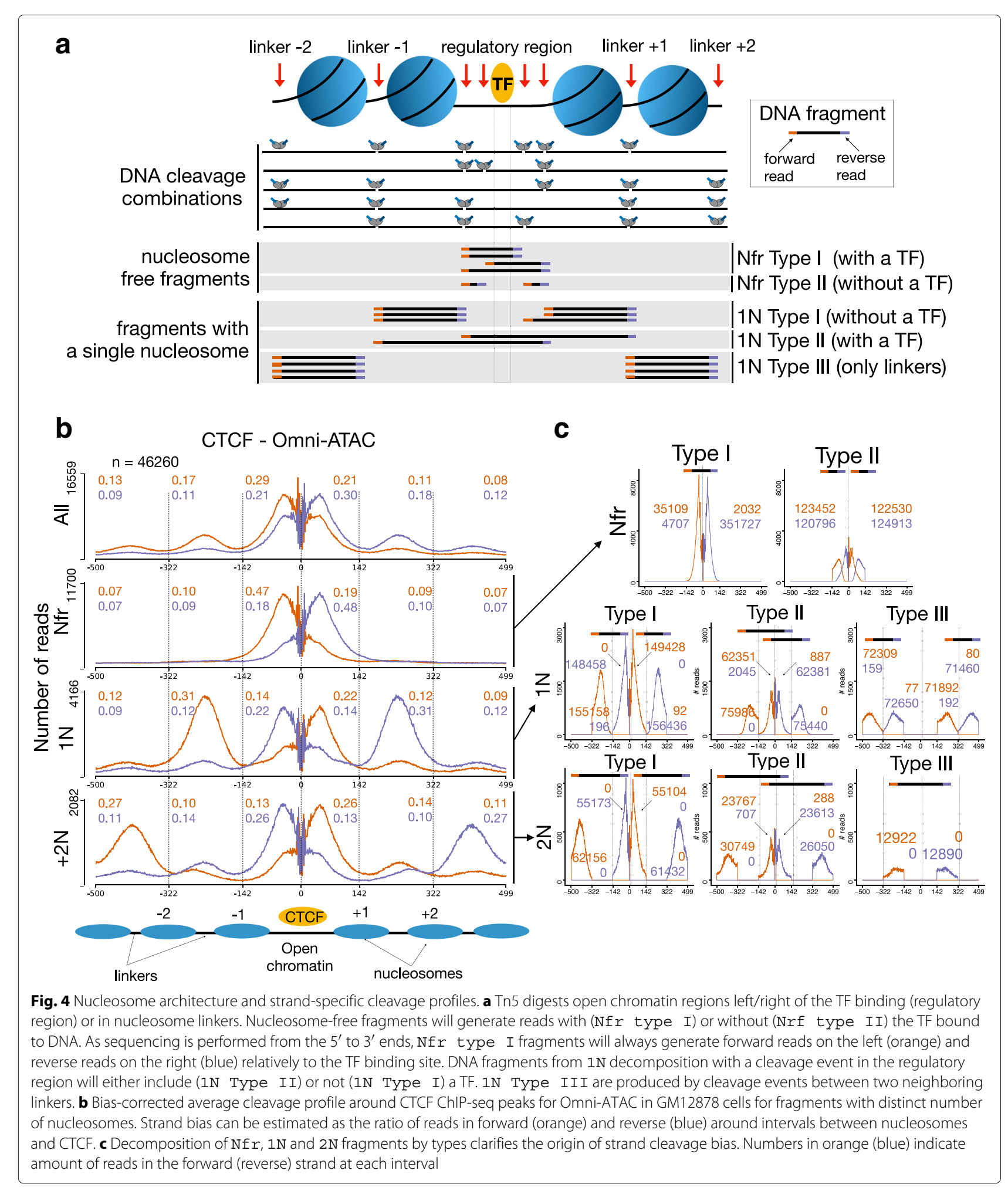

C

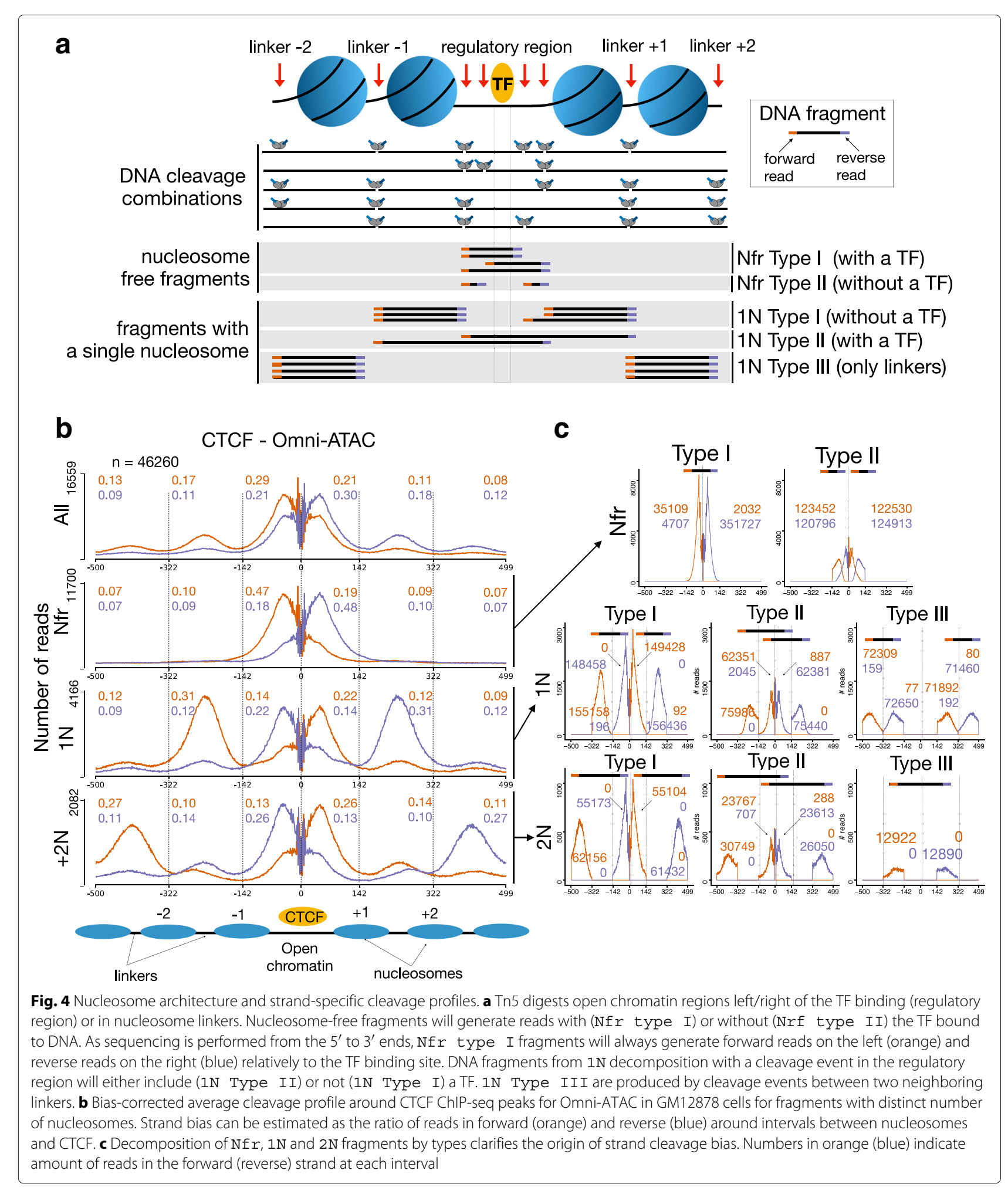
| $1 \mathrm{~N}$ Type I| (with a TF) $1 \mathrm{~N}$ Type III (only linkers)

considering all reads together (ratio of 1.38 for $A l 1$ ). We also observe high strand-specific bias in reads in linker regions, i.e., more forward reads in linkers -2 and -1 .

To further understand these observations, we separate reads by the types as proposed in Fig. $4 \mathrm{a}$ and count read frequencies in distinct regions (Fig. 4c). Considering $\mathrm{Nfr}$ fragments, we observe that Nfr type I generates almost exclusively forward reads left of CTCF. As sequencing is performed in the 5' to 3' direction, $\mathrm{Nfr}$ type I reads will only give rise to forward reads left 
of CTCF, while type II reads will generate both forward and reverse reads left of CTCF. Moreover, ATAC-seq protocols disfavor very short fragments associated to $\mathrm{Nfr}$ type II (Fig. 3b). These two facts cause the presence of a large number of forward reads from $\mathrm{Nfr}$ fragments left of the TF binding site. Following a similar rationale, $1 \mathrm{~N}$ type I fragments generate reverse strand reads left of CTCF, while $1 \mathrm{~N}$ type II fragments generate forward reads left of CTCF. There is a higher number of $1 \mathrm{~N}$ type I reads than $1 \mathrm{~N}$ type II reads, as ATAC-seq protocols bias disfavours too long fragments (Fig $3 \mathrm{~b}$ ). IN Type III reads are not relevant here, as their starting sites do not help the delineation of footprints. Equivalent patterns are also found in $+2 \mathrm{~N}$ reads. Similar strand bias for nucleosome decomposed signals are found in all ATAC-seq protocols and TFs (Additional file 1: Figure S17-S19), despite some variance in the distance between $\mathrm{TF}$ and linker regions [5].

\section{Comparative evaluation of footprinting methods in ATAC-seq data}

Next, we evaluate the performance of HINT-ATAC and state-of-the-art footprinting methods using an independent dataset based on K562 and H1-ESC cells (in total $148 \mathrm{TFs}$ ). We use three footprinting methods (DNase2TF, PIQ, and Wellington), which performed best in a recent comparative study based on DNase-seq data [8], and DeF$\mathrm{CoM}$, which was recently proposed for ATAC-seq data [15]. We have adapted Wellington and DeFCoM to evaluate them with PDM-based bias correction. As the baseline method, we include Tag Count (TC), which simply considers TFBSs inside peaks ranked by the number of reads after cleavage bias correction.

As before, methods are evaluated with the ranking scores, which combine AUPR and AUC values for distinct false positive rates for each TF. A higher ranking score indicates higher recovery of ChIP-seq supported footprints. HINT-ATAC is the top ranked method followed by Wellington and DeFCoM using PDM bias correction. HINT-ATAC has statistically significant higher ranking than all evaluated methods, and Wellington-PDM has statistically significant higher ranking than TC (Fig. 5a). One interesting question is the independent importance of (1) PDM bias correction and (2) nucleosome decomposition in HINT-ATAC performance. We observe that the independent use of PDM bias correction or nucleosome decomposition improves the performance of HINT, while neither improvement is significantly better than the other (Additional file 1: Figure S20).

Concerning competing methods, ranking of Wellington and DeFCoM is improved with the use of PDM bias correction, but we observe no clear improvement when using $\mathrm{N} f r$ reads (Additional file 1: Figure S20) ${ }^{3}$. The overall good performance of Wellington-PDM is likely due to its use of strand-specific signals. DeFCoM, which is based on a classifier, requires TF ChIP-seq data for training a model for each individual TF and is the only method requiring training on K562 and H1-ESC cells. Its performance is likely to decrease when training and predictions are performed across distinct cells. Taken together, these results confirm the fact that bias correction based on PDMs is crucial for prediction of ATAC-seq footprints and that HINT-ATAC has the best recovery of ChIP-seq supported footprints.

\section{Omni-ATAC-seq and DNase-seq are equivalent in the prediction of cell-specific transcription factor binding}

There is a perception in the field that DNase-seq libraries are superior to ATAC-seq for computational footprinting $[11,15]$. To address this, we compare the predictive performance of HINT-ATAC on distinct ATAC-seq protocols and DNase-seq of single-hit [31] and double-hit [32] protocols. We also analyze ATAC-seq experiments on distinct number of cells (bulk 50.000, 500, or single cells) [33]. To obtain a fair comparison, we first optimize HINT models for DNase-seq to consider strand-specific signals and the use of PDM-based correction (Additional file 1: Figure S13). Altogether, double-hit DNase-seq and Omni ATAC-seq are ranked best in all evaluated cells, while fast-ATAC-seq or ATAC-seq experiments based on 500 cells obtain the poorest results (Additional file 1: Figure S21). The lower performance of the former protocols is explained by their low quality indicators, i.e., fraction of reads insides peaks (FRIP) below 0.1 (Additional file 1: Figure S21).

Another limitation of ATAC-seq, which was previously discussed in the literature, is its lower coverage in enhancer regions [6,34]. To inspect if this also impacts on the prediction of footprints in enhancers, we divide ChIP-seq peaks as being in a promoter and enhancer regions using chromHMM annotations [35] and evaluate AUPR on both subsets. Indeed, we observe that footprints based on standard ATAC-seq have lower performance in enhancer regions relative to footprints based on DNaseseq data. However, no such difference is observed using Omni-ATAC-seq (Additional file 1: Figure S22). This indicates that improvements in the Omni-ATAC-seq protocol have a positive impact on the prediction of TFBSs in enhancers.

Finally, we inspect if one of the protocols is able to predict more accurately the binding of particular TFs. We observe that TFs with higher AUPR on ATAC-seq have more ATAC-seq cleavage sites surrounding the binding site and vice-versa, as exemplified by YY1 and JunD (Fig. 5b, c and Additional file 1: Figure S23). Interestingly, grouping of TFs by family indicates that DNase-seq obtains higher AUPR for bZIP and helix-loop-helix families (Fig. 5d and Additional file 1: Figure S24). This 


\section{a}

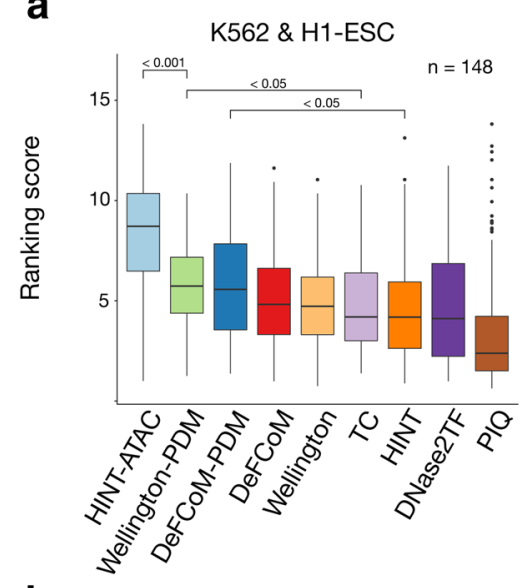

b

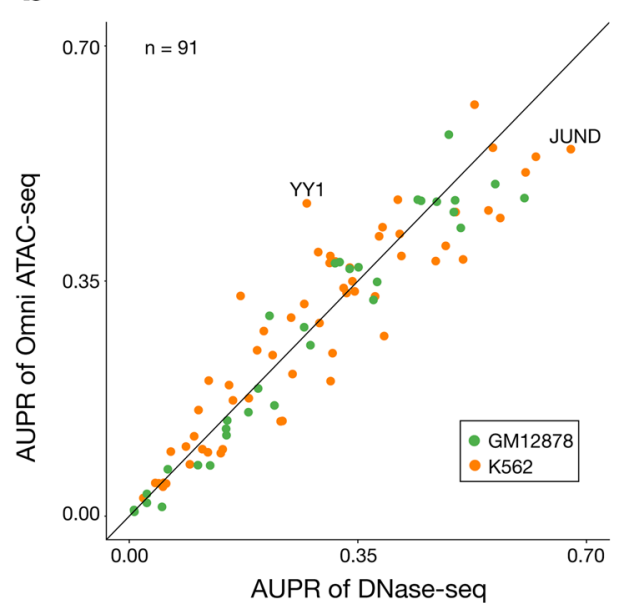

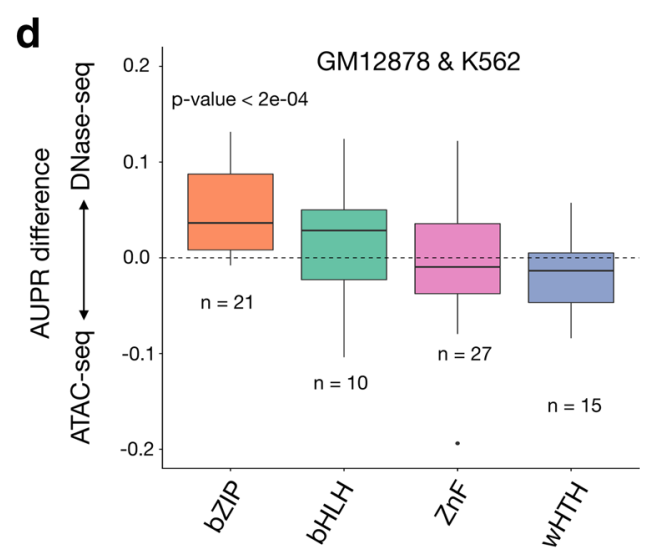

C

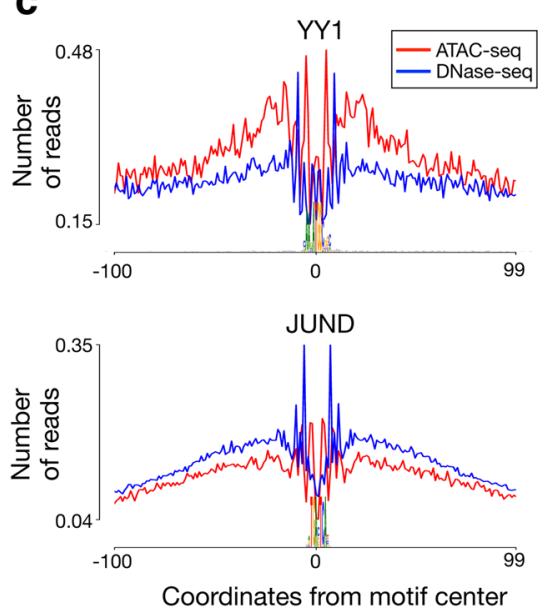

Fig. 5 Competing methods and protocols comparison. a Comparative evaluation of HINT-ATAC, HINT, Wellington, DNase2TF, DeFCoM, and PIQ on the test dataset (H1-ESC and K562 cells). A higher ranking score indicates highest recovery of ChIP-seq supported binding sites. $p$ values are based on the Friedman-Nemenyi test. We only show the significant $p$ values of the top 3 methods (see Additional file 1: Table S23-S24 for complete results). b AUPR values of DNase-seq (DH) vs ATAC-seq (Omni) for 91 factors, of which 41 factors obtain higher AUPR using ATAC-seq. c The footprint profiles of two factors with the highest AUPR difference are shown. $\mathbf{d}$ Difference in AUPR of double-hit DNase-seq and Omni ATAC-seq by grouping TFs by transcription factor families as defined in JASPAR database. Only families with more than 10 TFs are shown, and $p$ values are obtained with a $t$ test $($ mean $=0)$

suggests that structural features shared by TF families negatively affect $\operatorname{Tn} 5$ cleavage.

\section{HINT-ATAC finds relevant transcription factors for dendritic cell specification}

To demonstrate the performance of HINT-ATAC, we use HINT-ATAC footprints for detecting TFs in dendritic cells (DC), a specialized immune cell type involved in immunity and tolerance induction [36]. In short, we use a two-step culture system $[37,38]$ to differentiate multipotent progenitors (MPP) from mouse bone marrow into common DC progenitors (CDP). CDP are then further differentiated into classical DC type1 and type 2 (cDC1 and cDC2, respectively) or plasmacytoid DC (pDC) (Fig. 6a).
Cross-presenting $\mathrm{cDC} 1$ and $\mathrm{pDC}$ are particularly interesting and differ in specific immune functions, which is associated with subset specific gene expression repertoires. TFs are at the top of the hierarchy of gene expression networks driving cell identity and function and thus there is a particular interest in TFs in DC. We perform OmniATAC-seq experiments of $\mathrm{cDC} 1$ and $\mathrm{pDC}$ subsets and employ HINT-ATAC to detect footprints within ATACseq peaks for each of these two cell types. Next, we estimate changes in binding activity for 579 TFs with a motif in JASPAR [39]. Cell-specific TF activity is evaluated by measuring the depth of footprints and the total number of reads in flanking regions (see the "Method" section). Higher and lower differences in activity suggest that the 

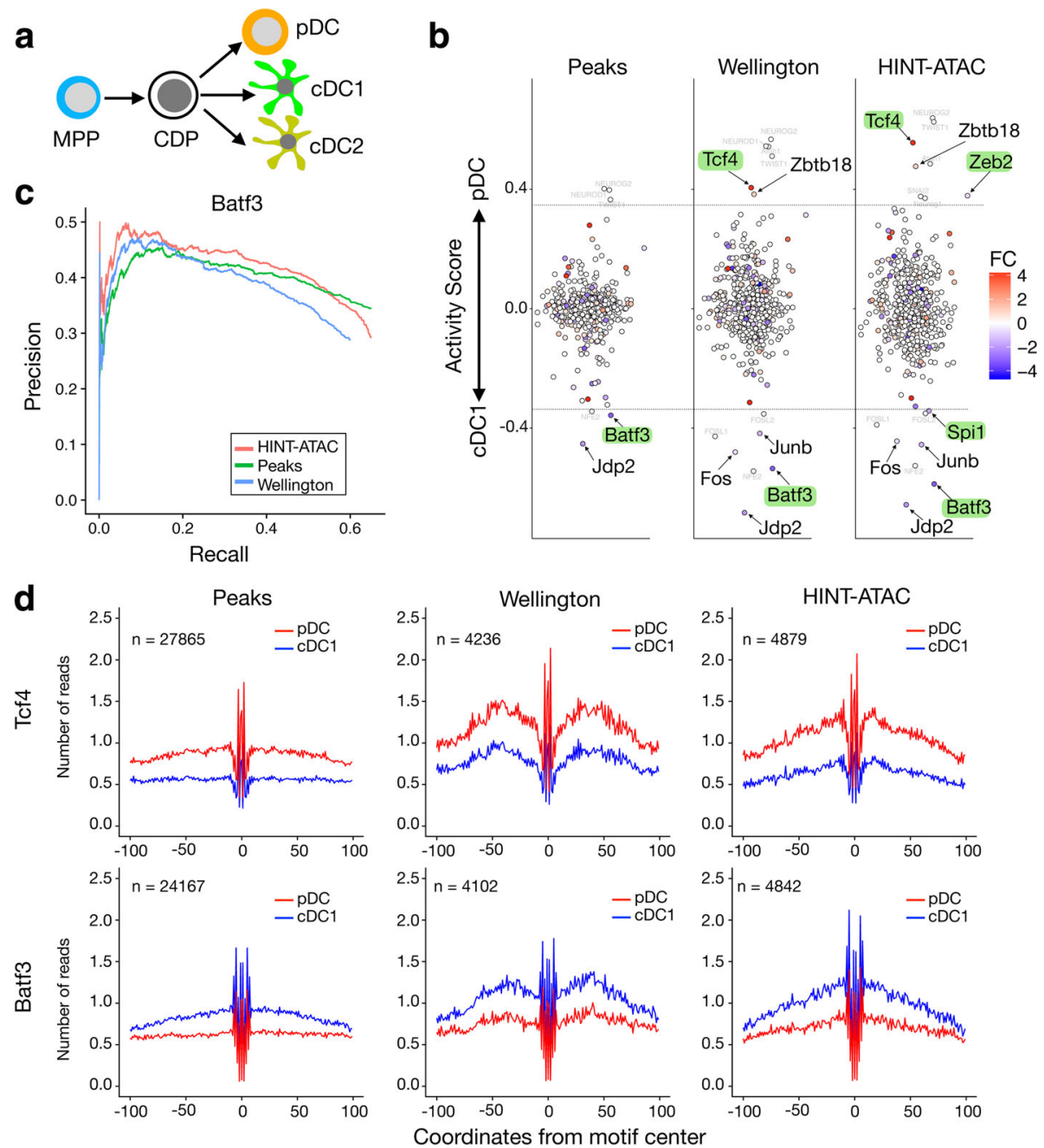

e

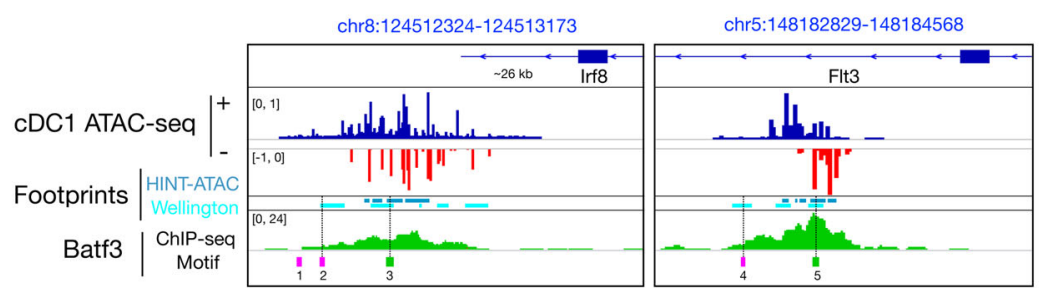

Fig. 6 Application to ATAC-seq data of dendritic cell (DC) differentiation. a A two-step culture system differentiates ex vivo multipotent progenitors (MPP) to DC progenitors (CDP) and further to classical DC type1 and type2 (CDC1 and CDC2, respectively) or plasmacytoid DC (pDC). b Cell-specific activity of 579 TFs with motifs in either ATAC-seq peaks or footprints by Wellington or HINT-ATAC. $Y$-axis indicates the difference in activity in CDC1 compared to pDC cells (pDC-cDC1). Names of TFs with significant differential activity values are shown (adjusted $p$ value $<0.05 ; t$ test) and represent TFs above/below dotted lines. TFs with at least $0.5 \mathrm{log}$ fold change (FC) in gene expression are highlighted (larger fonts), and known DC relevant TFs are marked in green. c Area under the precision recall curve evaluated with Batf3 ChIP-seq in cDC1. d Average cleavage profiles of Tcf4 and Batf3 motifs supported by ATAC-seq peaks, Wellington or HINT-ATAC footprints. e Regions with ATAC-seq and Batf3 ChIP-seq peaks in cDC cells close to DC relevant genes. We display all footprints from Wellington, HINT-ATAC, and all motifs found inside ATAC-seq peaks. While both Wellington and HINT-ATAC find footprints supporting motifs matching summits of Batf3 ChIP-seq peaks (sites 3 and 5 in green), Wellington footprints also support binding sites ( 2 and 4 in pink), which are not supported by the ChIP-seq signal

TF shows stronger binding in $\mathrm{pDC}$ and $\mathrm{cDC} 1$ cells, respectively. We also evaluate motifs supported by Wellington footprints or motifs inside ATAC-seq peaks ${ }^{4}$. We observe a number of TFs with statistically significant difference in activity between $\mathrm{cDC} 1$ and $\mathrm{pDC}$ (Fig 6b; $p$ value $<0.05 ; t$ test). We further filter TFs by only considering those with an absolute log2 fold change in gene expression higher than 0.5 . 
Interestingly, the most prominent $\mathrm{cDC} 1$ and $\mathrm{pDC}$ specific factors, identified by HINT-ATAC, represent two TFs shown to be important in these DC subsets: Batf3 [40] and Tcf4 [41]. Other known DC factors identified by HINT-ATAC include Zeb2, which was shown recently to be crucial in pDC differentiation [42], and Spi1 (also referred as PU1 or SFPI1), which is a master regulator of DC differentiation and mostly active in $\mathrm{CDC}$ [38]. The peak-based approach only detects significant activity changes for Batf3, while Wellington only predicted significant changes for Batf3 and Tcf4.

The higher precision of HINT-ATAC is also reflected in the higher AUPR for Batf3 (Fig. 6c), as supported by Batf3 ChIP-seq data in cDC1 from [40]. The average cleavage profiles for Batf3 and Tcf4 (Fig. 6d) and other selected TFs (Additional file 1: Figure S25) further exemplify this. The naive peak-based method is less specific and predicts five times more binding sites than footprinting methods. There is only a partial overlap between Wellington and HINT-ATAC footprints, i.e., $52.18 \%$ of footprints predicted by Welligton were also predicted by HINT-ATAC (footprints sharing at least $50 \%$ of bases). Notably, average cleavage profiles from Wellington had less signals in flanking regions than HINT-ATAC (Fig. 6d and Additional file 1: Figure S25). This is possibly due to Wellington's inability to define exact cleavage positions of the Tn5 enzyme. At individual regions, HINT-ATAC footprints are also more specific than other methods in detecting Batf3 binding sites, as exemplified in regions close to the DC relevant genes Irf8 [40] and Flt3 [38] (Fig. 6e).

\section{Discussion}

We demonstrate that the use of position dependency models is crucial for correction of cleavage bias for ATACseq and also improves correction for DNase-seq data. As shown in the subsampling experiments, $k$-mer-based estimates are less reliable than the PDMs for libraries with lower sequencing depth, as an indication of overfitting. Dependencies learned by PDMs for both Tn 5 and DNase-I are mostly based on adjacent positions. For Tn5, dependencies were detected between the middle of the Tn5 motif and positions 2 bps away. This might indicate a complex dependency of nucleotide recognition by the Tn5 dimer. These higher order dependencies might be associated with DNA shape, as recently demonstrated for DNase-I [29]. Previously, [11] reported difficulties in detecting footprints around motifs associated with regulatory variants in ATAC-seq data. We observed that PDM-based bias correction improves footprint profiles by increasing the footprint shapes for the majority of these motifs (Additional file 1: Figure S26), as an example of the caveats of ignoring cleavage bias.

There is growing attention in the field on cleavage biases present in sequencing protocols using cleavage/digestion enzymes [23, 43]. For example, the "digestion bias" of nucleases has been shown to induce artifacts in nascent RNA-seq protocols [43]. The same enzyme is used in ChIP-seq variants (ChIP-exo [44], ChIP-nexus [45]) and is likely to influence the detection of ChIP-exo footprints. PDMs represent a flexible framework for cleavage bias correction, which is likely to improve downstream analysis of any of these protocols.

The strand-specific cleavage patterns around transcription factor binding sites represent another overlooked aspect of ATAC-seq. Decomposition of DNA fragments by nucleosome number shows intricate strand specific cleavage patterns relative to TF binding. We demonstrate here that the strand bias of ATAC-seq protocols arises from the preference to particular cleavage events. Another evidence of the importance of neighboring proteins to $\operatorname{Tn} 5$ cleavage is the fact that the relative predictive power of ATAC-seq in relation to DNase-seq varies for particular TF families. This includes TFs from the bZIP and helixloop-helix families, which bind as dimers and have large structures. These structural properties are likely to impair access of $\operatorname{Tn} 5$ to neighboring DNA regions.

Footprints predicted in Omni-ATAC obtained better performance than standard and fast-ATAC protocols. Omni-ATAC libraries have higher fraction of fragments associated to mono and di-nucleosomes than standard or fast-ATAC protocols. This suggests that improvements introduced in Omni-ATAC protocol enrich for mono and bi nucleosome fragments, leaving more attenuated strand bias profiles in $1 \mathrm{~N}$ and $+2 \mathrm{~N}$ reads than standard or fastATAC-seq. While there is a perception of the field that a large number of reads are necessary for footprint predictions, libraries with moderate number of reads $(50$ millions) are among the best for ATAC-seq. On the other hand, the quality of the library, as indicated by the fraction of reads within peaks (FRIP), impacts the predictive power of footprints.

Finally, we show how footprints can be used to find TFs associated to DC subset specification. HINT-ATAC has the highest predictive power and identifies four TFs already associated with DC specification. Other predicted TFs with unknown (Zbtb18) or poorly understood (Jdp2) functions in DC development represent interesting candidates for future functional studies. A similar approach was used to identify TFs associated with regulation of beta cells in fasting vs. normal diets with DNase-seq [13]. This study considered all motifs inside DNase-seq peaks. As shown in our analysis, this simple strategy has lower power in detection of cell-specific TFs given the inclusion of a larger number of false positive binding sites.

\section{Conclusions}

We present here the first computational footprinting method tailored to the ATAC-seq protocol. HINT-ATAC 
corrects the cleavage bias of the Tn5 enzyme with a position dependency model and explores strand-specific bias, which is dictated by the local nucleosome architecture, to detect footprints. HINT-ATAC predictions outperform competing methods and have similar accuracy when applied to either Omni-ATAC-seq or DNase-seq protocols. This indicates that improvements in protocols and our computational approach make ATAC-seq a competitive alternative to DNase-seq for identifying TF binding sites, even for experiments based on moderate number of reads ( $\sim 50$ millions) and low number of cells $(\sim 25.000)$.

\section{Method}

\section{HINT-ATAC}

HINT is a computational framework for detection of footprints from open chromatin data [7]. It works in two major steps: first, genomic cleavage signals are generated from raw sequencing libraries after filtering reads by fragment size, correction of cleavage bias, and signal normalization. Next, cleavage signals are given as input to a HMM, which segments the signal and finds the location of footprints. HINT-ATAC extends HINT[7] by the proposal of a generalized framework for cleavage event counting and bias correction. This new framework allows cleavage events to be displaced from the read start and is based on a probability distribution assuming dependency between nucleotide positions. This allows to consider bias spanning larger genomic areas. HINT-ATAC also extends HINT by the use of strand-specific and fragment-size decomposition of cleavage signals as input. Finally, HINTATAC includes a novel semi-supervised training procedure, which uses a single TF ChIP-seq dataset for training. In contrast, the first version of HINT required the manual specification of the topology or data annotation for training [7]. Here, we will describe these novel aspects of HINT-ATAC.

\section{Cleavage event counting and correction of sequence bias} For a given open chromatin library and a reference genome sequence $G$ with length $N^{5}$, the first step is to generate the strand-specific genomic signals by counting the cleavage events on the positive or negative strands. HINTATAC considers the first position of aligned DNase-seq reads as the cleavage event as usual in the literature $[7,19]$. For ATAC-seq, the middle of the Tn 5 cleavage event is the fifth base after the fragment start (see Fig. 1a). Given an ATAC-seq read aligned with start position $i$, HINTATAC considers the position $i+4$ as a cleavage event for forward reads and $i-5$ for reverse reads ${ }^{6}$. This is equivalent to shifting positions of ATAC-seq reads as originally proposed in [5].

More formally, the strand-specific signals are defined by the vectors:

$$
\begin{aligned}
& \mathbf{y}^{+}=\left\langle y_{1}^{+}, \cdots, y_{i}^{+}, \cdots, y_{N}^{+}\right\rangle \\
& \mathbf{y}^{-}=\left\langle y_{1}^{-}, \cdots, y_{i}^{-}, \cdots, y_{N}^{-}\right\rangle,
\end{aligned}
$$

where $y_{i}^{+}\left(y_{i}^{-}\right)$indicates the number of cleavage events at a given genomic position $i$ at positive (negative) strands. In the case of paired end reads, it is possible to obtain the fragment length $\ell$, by considering the differences between left-most and right-most alignment positions of the read pair. HINT-ATAC also counts cleavage events by only considering reads in a particular fragment size range $(m<\ell \leq n)$, which we denote $\mathbf{y}^{+}(m, n)$. See below for definitions of intervals. In the following, we only consider the positive strand for simplicity.

Next, we correct the cleavage event profiles by sequence-specific cleavage bias considering the word $w[i]$ with size $k$ around genomic position $i$. For a given genome sequence $G$, this is defined as $w[i]=G[i-\lfloor k / 2\rfloor, i+$ $\lceil k / 2\rceil-1]$.

For an arbitrary word $w$, the bias is defined as

$$
b(w)=\frac{p(w \mid o b s)}{p(w \mid \exp )},
$$

where $p(w \mid$ obs $)$ is the probability of $k$-mer $w$ around the position of a cleavage event and $p(w \mid \exp )$ is the probability of finding word $w$ in the genome. For a position $i$ in the genome, the bias-corrected signal is obtained as [19]:

$$
x_{i}=\left(y_{i}+1\right) /\left(\hat{y}_{i} \cdot \hat{b}(w[i])+1\right),
$$

where $\hat{y}_{i}=\frac{1}{50} \sum_{j=i-25}^{i+24} y_{j}$ represents the average number of cleavage events around position $i$ and $\hat{b}(w[i])=$ $b(w[i]) / \sum_{j=i-25}^{i+24} b(w[j])$ is the bias of $w[i]$ normalized by the bias of surrounding genomic regions.

We describe below three distinct approaches to calculate cleavage bias estimates $b(w)$ : (i) the $k$-mer-based approach, which is widely used in the DNase-seq literature, (ii) a PWM-based approach, which is standard for modeling DNA-protein interactions, and (iii) our novel approach using PDMs.

\section{k-mer-based estimation}

The most common approach for bias estimation is to use the frequency of $k$-mers to estimate the probability $p(w)$ $[8,11,19,20,30]$. Let $W^{o b s}$ be a multiset with all words with length $k$ around individual cleavage events of an open chromatin library and freq( $w \mid o b s)$ be the frequency of a word $w$ in $W^{o b s}$. Then, the probability is estimated as

$$
p(w \mid o b s)=\frac{\text { freq }(w \mid o b s)}{\left|W^{o b s}\right|},
$$

where $\left|W^{o b s}\right|$ denotes the total number of $k$-mer occurences $\left|W^{o b s}\right|=\sum_{w}$ freq $(w \mid o b s)$. Similarly, $p(w \mid \exp )$ is estimated on the background multiset ( $W^{\exp }$ ). These estimates are plugged in Eq. 2 to obtain the final cleavage bias estimates. Equation 4 is equivalent to estimating a 
multinomial distribution of $k$-mers in multisets $W^{o b s}$ and $W^{\text {exp }}$. Estimates are prone to overfitting for large $k$ or low number of reads.

As standard in the field $[8,19]$, we define background regions as all accessible genomic regions, i.e., ATACseq or DNase-seq peaks of the corresponding library. An exception are naked DNA experiments, where the complete genome sequence is considered.

\section{PWM-based estimation}

An alternative approach, which is standard for transcription factor binding models [46], is the use of models assuming independence between positions of $w$. That is,

$$
p(w \mid o b s)=\prod_{j=1}^{k} p\left(w_{j}=b \mid o b s\right),
$$

where $w_{j}$ is the $j$ th position at word $w$ and $b \in\{A, C, G, T\}$ a genomic base. We define freq $_{j}(b \mid o b s)$ as the frequency of base $b$ to occur in position $j$ in all words in multiset $W^{o b s}$. Then, we can estimate the probability as

$$
p\left(w_{j}=b \mid o b s\right)=\frac{\text { freq }_{j}(b \mid o b s)}{\left|W^{o b s}\right|} .
$$

Estimates for $p(w \mid \exp )$ are calculated similarly from the background multiset $W^{\exp }$.

\section{PDM-based estimation}

A main disadvantage of PWM-based estimation is that it assumes statistical independence between different positions in $w$. An alternative between models considering all dependencies (k-mer approach) and no dependencies (PWM approach) is provided by a position dependency model (PDM). PDMs consider dependencies between particular pairs of positions $j$ and $l$ up to a particular distance $d$, i.e., $d \geq|l-j|$ and $l<j$. We propose here the use of a special class of PDMs (SLIM models) [26] to estimate the probability of $w$, that is

$$
p(w)=\prod_{j=1}^{k}\left(p\left(C_{j}=0\right) \cdot p\left(w_{j}\right)+p\left(C_{j}=1\right) \sum_{\substack{l \in[1, k] \\ j-l \leq d}} p\left(R_{l j}\right) \cdot p\left(w_{j} \mid w_{l}\right)\right),
$$

where $p\left(C_{j}\right)$ is the prior probability that the distribution at position $j$ should be modeled conditional on other positions; $p\left(R_{l j}\right)$ is the prior probability that position $j$ should be conditional on position $l ; p\left(w_{j}\right)$ is the probability of a base to appear in position $j$ and $p\left(w_{j} \mid w_{l}\right)$ is the probability of base in position $j$ conditional on the base from position $l$. It is worth noting that the PWM-based estimation is a special case of SLIM models with $p\left(C_{i}=0\right)=1$ for all positions.

For a given multiset $W$, estimates $p\left(w_{j}\right)$ follow Eq. 6 . Conditional estimates $p\left(w_{j} \mid w_{l}\right)$ can be derived analogously using frequencies of bi-nucleotides found in positions and $l$ in a multiset. The missing estimates $p\left(C_{j}\right)$ and $p\left(R_{l j}\right)$ are obtained with a discriminative maximum supervised posterior principle, which uses random sequences as negative models, see [26] for more details. These methods are applied on multisets $W^{o b s}$ and $W^{\exp }$ to obtain $p(w \mid o b s)$ and $p(w \mid \exp )$, which are then used as final bias estimates with Eq. 2. In our experiments, we only consider dependencies such that $d<6$.

\section{Strand and nucleosome number decomposition and signal post-processing}

For paired-end ATAC-seq libraries, we also filter signals by only considering cleavage events from paired-ends with a particular size range $\left(\mathbf{y}^{+}(m, n)\right)$. We define distinct fragment size intervals with contain distinct number of nucleosomes by estimating local minima between modes of the fragment size distribution of the ATAC-seq library used for model training (Additional file 1: Figure S11). For standard ATAC-seq, the first interval $(0,145]$ represents nucleosome-free reads ( $\mathrm{Nfr})$, the interval $(145,307]$ represents one nucleosome reads $(1 \mathrm{~N})$, the interval $(145, \infty]$ represents one or more nucleosome reads $(+1 \mathrm{~N})$ and the interval $(307, \infty]$ represents two or more nucleosomes $(+2 \mathrm{~N})$. We then evaluate distinct strategies with combinations of these signals: all reads ( $A 11)$, signal from nucleosome-free reads ( $\mathrm{Nfr}$ ), signals from nucleosomefree reads and signals from reads with one or more nucleosomes ( $\mathrm{Nfr} \&+1 \mathrm{~N})$, and signals from nucleosome free, signals from one nucleosome, and signals from one or more nucleosomes $(\mathrm{Nfr} \& 1 \mathrm{~N} \&+2 \mathrm{~N})$.

For each decomposition strategy, we apply the cleavage bias correction, a within-signal normalization by averaging non-zero counts inside bins and a between-signal normalization by applying a logistic function. To estimate the slope of the signals, we apply a Savitzky-Golay smoothing filter by fitting the data into a second order polynomial and performing a convolution (based on a specific window length) with a vector containing Savitzky-Golay coefficients. Normalization steps are described in details in [7].

For example, in the case of All cleavage events with strand specific signals, we have a signal and a slope value for either positive and negative strands, that is:

$$
X=\left\{x_{\text {norm }}^{+}, x_{\text {slope }}^{+}, x_{\text {norm }}^{-}, x_{\text {slope }}^{-}\right\} \text {. }
$$

\section{HMM training and decoding}

We take the previously described multivariate cleavage signals $X$ as input for the HMM model. The HMM contains a multivariate normal density function with full covariance matrix as emission probability for each state. The dimension depends on the decomposition strategy and varies from 2 (non-strand specific) 
to 12 dimensions (strand-specific models considering 3 distinct fragment size intervals). For a given TF, we obtain regions with ChIP-seq peaks and a motif predicted binding site (MPBS) as described in the "Evaluation of footprinting prediction" section. We then estimate the average ATAC-seq profile for the region $\pm 500 \mathrm{bp}$ to the motif center and annotate the center with the label FOOTPRINT. Next, we use a fully connected HMM with $S$ states and select one state to represent the FOOTPRINT. We use a semi-supervised algorithm to train the HMM [47]. This algorithm learns the parameters of the HMM in a supervised manner for the FOOTPRINT state and using the Baum-Welch algorithm for all other $S-1$ states (see Additional file 1: HMM Training). The initial model parameters are obtained after execution of a M-step with random posterior distributions with the exception of the FOOTPRINT state, which has the posterior distribution defined by the labels.

To detect footprints in a novel sequencing library, we use the Viterbi algorithm [49] to find the most probable sequence. We consider positions annotated with the FOOTPRINT state to be the active TFBS. This approach has clear advantages over previous HMM-based footprinting methods $[7,8,50]$, which require the manual specification of a HMM topology and manual annotation of training data to estimate models. We have evaluated the use of different ChIP-seq datasets of several factors for GM12878 cells for training (CTCF, EGR1, SP1, USF2 and ZNF143). We observed no statistical differences between the models. Thus in order to simplify experimental design, we have arbitrarily selected the model based on EGR1 ChIP-seq in GM12878 cells. The model was employed in all further experiments including ATAC-seq from other cells. EGR1 ChIP-seq was excluded from any evaluation. The number of states $S$ can also be varied and will be therefore optimized for each protocol and signal decomposition.

\section{Cell-specific TF activity}

We propose here a simple statistic (activity score-ACT) to measure the strength of TF binding in a particular biological condition. First, we identify all binding sites of a particular TF overlapping with footprints $F=$ $\left\{\left(f_{1}^{l}, f_{1}^{r}\right), \ldots,\left(f_{n}^{l}, f_{n}^{r}\right)\right\}$, where $f^{l}$ and $f^{r}$ represent the leftmost and rightmost genomic positions of the binding site. The activity score of a TF is defined as

$$
A C T(T F)=\frac{1}{|F|} \sum_{\left(f^{l} f^{r}\right) \in F}\left(\frac{1}{2} \sum_{j=f^{l}-e-1}^{f_{i}-1} x_{j}+\frac{1}{2} \sum_{j=f^{r}+1}^{f^{r}+e+1} x_{j}-\sum_{j=f^{l}}^{f^{r}} x_{j}\right)+\left(\sum_{j=f^{l}-100}^{f^{r}+100} x_{j}\right),
$$

where $e$ is the length of the binding site and $x_{j}$ is the cleavage event signal (after bias correction) at genomic position $j$.

The activity score can be seen as a combination of the protection score [8], which measures the difference in cleavage events between the footprint and flanking regions (left term), and the openness score [51], which simply measures the number of cleavage events around the binding site (right term).

Here, we are interested in identifying TFs with change in activity between two conditions. This is given by the difference in ACT scores between two biological conditions, this is:

$$
\triangle A C T(T F)=\frac{A C T_{2}(T F)}{\omega_{2}}-\frac{A C T_{1}(T F)}{\omega_{1}},
$$

where $\omega_{1}$ and $\omega_{2}$ are normalization factors based on median-of-ratios [52]. These factors correct for differences of sequencing depths of libraries of the two conditions. Here, the set $F$ corresponding to binding sites supported by footprints in at least one of the conditions. We use this score to rank TFs with a known motif, where highest $\triangle A C T(T F)$ indicate TFs with specific binding in condition 2 .

\section{Materials and experimental design Low level analysis of DNase-seq and ATAC-seq libraries}

We used ATAC-seq data of cell lines GM12878 and K562 from $[5,6,28,53]$ and single-cell ATAC-seq data from cell lines GM12878, K562, H1-ESC [33], mouse blood cells from [54], and DNase-seq data of GM12878, K562, and H1-ESC from [55]. We also used naked DNA ATAC-seq data from [21] and naked DNA DNase-seq data from [30] for estimation of cleavage bias.

First, adapter sequences were trimmed from FastQ files using Trim Galore [56] with the following settings (-q 30 -paired -trim1). Reads were mapped to the reference genome using Bowtie2 [57] with the following parameters (-X2000 -no-mixed -no-discordant) allowing fragments of up to $2 \mathrm{~kb}$ to align. Duplicates were removed and reads were filtered for alignment quality of $>$ Q30 using samtools [58]. Next, MACS2 [59] was used to call ATACseq or DNase-seq peaks with the following parameters (-nomodel -nolambda -keep-dup auto -call-summits). The overlapping peaks were merged and then filtered for q-value $>10$. The same preprocessing was applied to naked DNA ATAC-seq and DNase-seq except for peak calling. All organism-specific data are based on human genome build 37 (hg19) and mm10. Reads mapping to the mitochondria, unmapped contigs and chromosome $\mathrm{Y}$ were removed from all subsequent analyses. Concerning ATAC-seq experiments performed on single cells, we combined all sequence libraries to consider them as a 
bulk experiment. See Additional file 2 for complete list of libraries and quality statistics.

\section{ATAC-seq on dendritic cell specification}

Dendritic cells (DC) are professional antigen presenting cells that comprise different subsets: classical DC type1 and type 2 ( $\mathrm{cDC} 1$ and $\mathrm{cDC} 2$, respectively) and plasmacytoid $\mathrm{DC}(\mathrm{pDC})$. In this study $\mathrm{cDC} 1$ or $\mathrm{pDC}$ were obtained in a two-step in vitro culture system according to [37]. Briefly, mouse bone marrow cells were first amplified with a specific cytokine cocktail and then induced to differentiate into DC with Flt3 ligand. $\mathrm{cDC} 1$ are CD11c+ CD11b+ $\mathrm{XCR} 1+$ and $\mathrm{pDC}$ are CD11c+ CD11b- B220+ and thus $\mathrm{cDC} 1$ and $\mathrm{pDC}$ subsets were purified by FACS sorting and subjected to Omin-ATAC-seq analysis. Omni-ATAC-seq was performed according to [6] with minor modifications. Prior to transposition dead cells were removed by centrifugation $\left(800 \mathrm{rpm}, 4 \mathrm{~min}, 4{ }^{\circ} \mathrm{C}\right)$. The transposition reaction was with $7.5 \mu \mathrm{L}$ Tagment DNA Enzyme 1 (TDE1) for $60 ' \mathrm{~min}$ at $37{ }^{\circ} \mathrm{C}$. Pre-amplification was with NEBNext Ultra II Q5 Master Mix and Nextera PCR Primers (5 cycles). Quantitative PCR amplification was with NEBNext Ultra II Q5 Master Mix, Nextera PCR Primer and SYBR Gold to determine the number of additional cycles. PCR amplification of additional cycles was as for pre-amplification. PCR fragments were purified with Qiagen MinElute PCR Purification Kit and library concentration and quality were determined by Agilent High Sensitive DNA Kit and Bioanalyzer, respectively. ATACseq libraries were sequenced with a Illumina NextSeq 500 Platform with 75 bps paired-end reads in duplicates. Trimming, alignment (to mouse genome mm9), and peak calling were performed as for other ATAC-seq libraries. Replicate libraries were merged previously to footprinting.

\section{Evaluation of footprinting prediction}

ChIP-seq of TFs and motif-predicted binding sites (MPBSs) were used as ground truth to evaluate the footprinting prediction in this work following $[8,27]$. We used here peaks from 124 TF ChIP-seq datasets provided by the ENCODE analysis working group on cell lines K562 (60), H1-ESC (31), and GM12878 (33) [60]. We obtained a PWM for each factor from the Jaspar database [61]. For a few exceptional cases were a motif was not found in Jaspar (5 TFs), we used matrices from Uniprobe [62], or Transfac [63]. See Additional file 1: Table S31 for stastistics of ChIP-seq data and motifs. Next, we used a motif matching tool based on the MOODS C++ library [64] to find MPBS. We determined a bit-score cut-off threshold by applying the dynamic programming approach described by [65] with an FPR of $10^{-4}$. Then, we created labels by combining MBPSs with ChIP-seq data for every TF. Specifically, MPBSs with ChIP-seq evidence (MPBS located within $100 \mathrm{bp}$ from the ChIP-seq peak summit) are considered true binding sites and MPBSs without ChIP-seq evidence are considered false binding sites. Footprint/MPBS pairs supported by ChIP-seq peaks are considered true positives (TP), while footprints with no ChIP-seq support are considered false positives (FP). TN and FN are defined accordingly. We rank the predictions for all methods by tag count (TC) as this has been shown to be the best method for ranking predictions [8].

To assess the accuracy, we created receiver operating characteristic (ROC) curves and evaluated area under ROC at $100 \%, 10 \%$, and $1 \%$ FPR by using the contingency table (TPs, FPs, TNs, and FNs). We also measured the area under Precision-Recall (auPRC) at 100\%, 10\%, and $1 \%$ recall as these measures are more suitable for data with skew on negative classes $[66,67]$. As the relative performance of methods might vary on distinct evaluation measures, we combine these with the approach used in the ENCODE-DREAM Challenge (https://www.synapse. org/\#!Synapse:syn6131484/wiki/405275). This score is equal to the sum over all six normalized ranking measures of $-\log (r /(N+1))$ where $\mathrm{r}$ is the rank of an algorithm for a specific performance measure (e.g., auROC) and $\mathrm{N}$ is the total number of methods. Therefore, best measures should have a high ranking in several of the evaluated measures.

\section{Evaluation data sets}

We divided our evaluation data in three distinct sets. The first set (training dataset) is composed of $32 \mathrm{TFs}$ ChIP-seq from the GM12878 and different ATAC/DNaseseq data. To evaluate bias correction methods, we used ATAC-seq data from standard protocol based on 50.000 cells [5], Omni protocol [6], Fast protocol [28], and naked DNA [21]. We also used DNase-seq (single hit) of the same cell line from ENCODE and naked DNase-seq from [30] to evaluate the impact of novel bias correction strategies on DNase-seq data. In addition, we included doublehit DNase-seq of GM12878 to evaluate HMM learning strategies. The next data set (test dataset) is based on 148 combinations of TF ChIP-seq and ATAC-seq experiments from H1-ESC and K562 cells. This data set was used to compare HINT-ATAC with other footprinting methods. ATAC-seq data K562 from 50.000 cells from [14] and combined single cell ATAC-seq data from H1ESC and K562 cells [33] and Omni-ATAC K562 cells from [6]. In above analyses, we excluded the TF ChIP-seq data from EGR1, which was used as label for the model (see Additional file 2 for full description of ATAC/DNase-seq data).

Finally, for the comparative evaluation of ATAC-seq and DNase-seq protocols, we defined a comprehensive data set by combining all TF-ChIP data (124) from previous data sets. This dataset is further enhanced by using single cell ATAC-seq libraries (GM12827, K562). 
We also annotated transcription factors regarding their structural family in accordance to JASPAR [39]. For statistical power, we only kept families with more than 10 annotated TFs. Concerning the comparison of footprinting performance on enhancer versus promoter regions, we obtained histone based segmentation of the corresponding cells with chromHMM [35] for GM1878 and K562 cells. We combined all states associated with enhancer or promoter regions which were used to split our TFBS prediction sets into two (Additional file 1: Table S31).

Statistical comparison of computational methods regarding TF binding sites was performed with the non-parametric Friedman-Nemenyi hypothesis test. Such a test provides a rank of the methods as well as the statistical significance of the out-performance of a particular method. Comparisons based on non-paired distributions were performed with the Wilcoxon rank sum test. All reported $p$ values based on multi-comparison tests were corrected using the Benjamini-Hochberg method.

\section{Endnotes}

${ }^{1}$ Query performed using the words "ATAC-seq" and "DNase-seq" at August 16, 2018 considering the number of series and samples deposited within the last year

${ }^{2}$ For each cleavage signal, HINT-ATAC also generates a slope signal to help detection of regions with increase/decrease on cleavage sites

${ }^{3}$ Given the fact Wellington and DeFCoM do not support multivariate signals, it is only possible to evaluate the Nfr decomposition for these methods.

${ }^{4}$ DeFCoM cannot be used in this unbiased analysis as it requires TF ChIP-seq data to train models, which is not given for all 579 TFs deposited in JASPAR.

${ }^{5}$ For sake of simplicity, we assume genomes are formed as a single chromosome.

${ }^{6} \mathrm{An}$ additional base pair is required to define the position of a cleavage event from reads mapped to negative strand ( -5 instead of -4$)$, as the right most positions of genomic intervals include an extra base.

\section{Additional files}

Additional file 1: Supplementary methods, figures and tables. (PDF 6,084 kb) Additional file 2: Description of ATAC-seq and DNase-seq data. (XLSX $19 \mathrm{~kb}$ )

\section{Acknowledgements}

We would like to thank Eduardo Gade Gusmao and Alvaro Rada-Iglesias (Centre for Molecular Medicine Cologne, CMMC, Cologne, Germany) for discussions; and Jens Keilwagen (Julius Kühn-Institut, Quedlinburg, Germany) for support with the SLIM model code. We also thank Katrin Götz (Department of Cell Biology, Institute for Biomedical Engineering, RWTH Aachen University Medical
School, Aachen, Germany) for cell sorting; Bernd Denecke and Jasmin Hübner (Interdisciplinary Center for Clinical Research (IZKF) Aachen Genomics Facility, RWTH Aachen University Medical School, Aachen, Germany) for sequencing.

\section{Funding}

This work was in part funded by the Interdisciplinary Center for Clinical Research (IZKF) Aachen, RWTH Aachen University Medical School, Aachen, Germany and by the Deutsche Forschungsgemeinschaft (DFG-GE 2811/3). Simulations were performed with computing resources granted by ITC RWTH Aachen University under project rwth0233.

\section{Availability of data and materials}

HINT-ATAC is an open source software and available online at http://www. github.com/CostaLab/reg-gen as part of the Regulatory Genomic Toolbox (RGT) under GNU General Public License v3.0. It is also available in Zenodo with the following DOI: 10.5281/zenodo.2540828 [68]. RGT and HINT-ATAC includes methods for motif matching, generation of average profiles and differential footprint analysis. Examples of use, including some of the analysis of this work, are provided as tutorials (http://www.regulatory-genomics.org/hint/tutorial).

This work generated novel OMNI ATAC-seq data on dendritic cell specification. This data is deposited at Gene Expression Omnibus (GEO) (GSE1 18221) [69]. The ATAC-seq data for GM12878 and K562 cells using standard protocol were obtained from GSE47753 and GSE70482 [53]; single cell ATAC-seq data for H1-ESC were obtained from GSE65360 [33]; and ATAC-seq data for mouse and human blood cells were obtained from GSE60103 [54] and GSE74912 [28]. Omni-ATAC and Fast-ATAC data for GM12878 and K562 cells were obtained from Sequencing Read Archive (SRA) with the BioProject accession PRJNA380283 [6]. DNase-seq data for K562, GM12878, H1-ESC cells using single-hit protocol were obtained from GSE32970 [55], and using double-hit protocol were obtained from GSE29692 [55]; and DNase-seq data with naked DNA were obtained from GSE61105 [30].

The TF Chip-seq enriched regions (peaks and summits) were obtained from ENCODE project [60] under the following experiment IDs: ENCSRO00EFY [70], ENCSROOODNZ [71], ENCSROOODOG [72], ENCSROOOEGD [73], ENCSRO0OEGV

[74], ENCSROOODOA [75], ENCSROOOEHE [76], ENCSROOOEGM [77], ENCSROOOBNK [78], ENCSRO00EWL [79], ENCSRO00EWJ [80], ENCSRO00DKB

[81], ENCSROOODKA [82], ENCSROOOBNE [83], ENCSRO00DJY [84], ENCSRO0ODJX [85], ENCSROOOBMD [86], ENCSROOOEFU [87], ENCSROOOBKQ [88], ENCSROOOFAI [89], ENCSRO0OBMV [90], ENCSRO0OBLO [91], ENCSRO0OEWM [92],

ENCSRO0OEWG [93], ENCSRO0OEGL [94], ENCSROOOFAH [95], ENCSRO0OEGN [96], ENCSROOOEGI [97], ENCSRO0OEGX [98], ENCSRO00EFV [99], ENCSROOOBNV [100], ENCSROOOFAG [101], ENCSROOOFAF [102], ENCSROOOEGR [103], ENCSROOOEGQ [1 04], ENCSROOOBRS [105], ENCSRO0OEHH [1 06], ENCSROOOBGW [107], ENCSROOOFAD [108], ENCSROOOBMW [109], ENCSROOOEGO [110], ENCSROOOBGX [1 1 1 ], ENCSROOOEGW [112], ENCSROOOBKO [113], ENCSROOOBNL [114], ENCSROOOBLK [115], ENCSROOOFAU [116], ENCSROOOFBC [117], ENCSROOOBRR [1 18], ENCSROOOEHB [119], ENCSROOOEHA [120], ENCSROOOBNN [121], ENCSROOOEWH [122], ENCSROOOBKT [123], ENCSROOOEHG [124], ENCSROOOEWF [125], ENCSROOOBKF [126], ENCSROOOBME [127], ENCSROOOEGP [128], ENCSROOOEWN [129], ENCSROOOBJY [130], ENCSROOODZJ [131], ENCSRO0OBRX [132], ENCSROOODZN [133], ENCSRO0ODYY [134], ENCSRO00BRG [135], ENCSROOOBMB [136], ENCSROOODZB [137], ENCSRO00BKA [138], ENCSROOOEYZ [139], ENCSROOOEYV [140], ENCSROOODZF [141], ENCSRO0OBKB [142], ENCSROOODKU [143], ENCSROOODZY [144], ENCSROOODNN [145], ENCSROOODNM [146], ENCSROOODZO [147], ENCSROOOBQS [148], ENCSROOOBGQ [149], ENCSRO0OEAC [150], ENCSRO0ODZW [151], ENCSRO0OBJE [152], ENCSRO0ODZP [153], ENCSRO0OBHK [154], ENCSRO00BGE [155], ENCSROOODZM [156], ENCSROOOBQZ [157], ENCSRO0OEUL [158], ENCSROOODZU [159], ENCSRO0OEUM [160], ENCSRO0OBHC [161], ENCSROOODZL [162], ENCSROOOBKC [163], ENCSROOOEBQ [164], ENCSRO0OEBX [165], ENCSROOOEBV [166], ENCSRO0OBNH [167], ENCSRO0OBJA [168], ENCSROOOBNS [169], ENCSROOOBIW [170], ENCSRO00ECA [171], ENCSRO0OEBZ [172], ENCSROOOEBS [173], ENCSROOOEUP [174], ENCSROOOEBY [175], ENCSROOOECC [176], ENCSRO0OBKK [177], ENCSROOOBMU [178], ENCSRO00ECE [179], ENCSROOOBHM [180], ENCSROOOECF [181], ENCSROOOBJW [182], ENCSROOOBIQ [183], ENCSROOOBIR [184], ENCSROOOBQG [185], ENCSRO0OBQV [186], ENCSROOOBIV [187], ENCSROOOECB [188], ENCSROOOBIT [189], ENCSRO0OBIU [190], ENCSRO00ECD [191], ENCSRO0OBKD [192], ENCSRO00EBW [193]. DNase-seq and ATAC-seq libraries are listed in a tabular form in Additional file 2, while TF ChIP-seq experiments are listed in Additional file 1: Table S31. 


\section{Authors' contributions}

IC coordinated and conceived this study with the support from MS and MZ. ZL performed the computational work, and TL and MB performed ATAC-seq experiments on dendritic cells. ZL and IC wrote the manuscript with feedback from all authors. All authors read and approved the final manuscript.

\section{Ethics approval and consent to participate}

Not applicable.

\section{Consent for publication}

Not applicable.

\section{Competing interests}

The authors declare that they have no competing interests.

\section{Publisher's Note}

Springer Nature remains neutral with regard to jurisdictional claims in published maps and institutional affiliations.

\section{Author details}

${ }^{1}$ Institute for Computational Genomics, Joint Research Center for Computational Biomedicine, RWTH Aachen University Medical School, 52074 Aachen, Germany. ${ }^{2}$ Department of Cell Biology, Institute of Biomedical Engineering, RWTH Aachen University Medical School, 52074 Aachen, Germany. ${ }^{3}$ Cluster of Excellence for Multimodal Computing and Interaction, Saarland Informatics Campus, Saarland University, Saarbrücken, Germany. ${ }^{4}$ Computational Biology \& Applied Algorithmics, Max Planck Institute for Informatics, Saarbrücken, Germany. ${ }^{5}$ Institute for Cardiovascular Regeneration, Goethe University, Frankfurt am Main, Germany. ${ }^{6}$ German Centre for Cardiovascular Research (DZHK), Partner site RheinMain, Frankfurt am Main, Germany. ${ }^{7}$ Helmholtz Institute for Biomedical Engineering, RWTH Aachen University, Aachen, Germany. ${ }^{8}$ Institute of Human Genetics, RWTH Aachen University Medical School, Aachen, Germany.

Received: 13 February 2018 Accepted: 25 January 2019

Published online: 26 February 2019

\section{References}

1. Boyle AP, Davis S, Shulha HP, Meltzer P, Margulies EH, Weng Z, Furey TS, Crawford GE. High-resolution mapping and characterization of open chromatin across the genome. Cell. 2008;132(2):311-22.

2. Crawford GE, Holt IE, Whittle J, Webb BD, Tai D, Davis S, Margulies EH, Chen Y, Bernat JA, Ginsburg D, et al. Genome Res. 2006;16(1):123-31.

3. Neph S, Stergachis AB, Reynolds A, Sandstrom R, Borenstein E, Stamatoyannopoulos JA. Cell. 2012;150(6):1274-86.

4. Vierstra J, Stamatoyannopoulos JA. Nat Methods. 2016;13(3):213-21.

5. Buenrostro JD, Giresi PG, Zaba LC, Chang HY, Greenleaf WJ. Nat Methods. 2013;10(12):1213-8.

6. Corces MR, Trevino AE, Hamilton EG, Greenside PG, Sinnott-Armstrong Na, Vesuna S, Satpathy AT, Rubin AJ, Montine KS, Wu B, Kathiria A Cho SW, Mumbach MR, Carter AC, Kasowski M, Orloff La, Risca VI, Kundaje A, Khavari Pa, Montine TJ, Greenleaf WJ, Chang HY. An improved ATAC-seq protocol reduces background and enables interrogation of frozen tissues. Nat Methods. 2017. 14(10): 959-62 https://doi.org/101038/nmeth4396.

7. Gusmao EG, Dieterich C, Zenke M, Costa IG. Bioinformatics. 2014;30(22):3143-51.

8. Gusmao EG, Allhoff M, Zenke M, Costa IG. Nat Methods. 2016;13(4): 303-9.

9. Neph S, Vierstra J, Stergachis AB, Reynolds AP, Haugen E, Vernot B, Thurman RE, John S, Sandstrom R, Johnson AK, et al. Nature. 2012;489(7414):83-90.

10. Schmidt F, Gasparoni N, Gasparoni G, Gianmoena K, Cadenas C, Polansky JK, Ebert P, Nordström K, Barann M, Sinha A, et al. Nucleic Acids Res. 2017:45(1):54-66.

11. Schwessinger R, Suciu MC, McGowan SJ, Telenius J, Taylor S, Higgs DR, Hughes JR. Genome Res. 2017;27(10):1730-42. https://doi.org/ 101101/gr220202117.

12. Kolovos P, Georgomanolis $T$, Koeferle A, Larkin JD, Brant L, Nikolicć M, Gusmao EG, Zirkel A, Knoch TA, van ljcken WF, Cook PR, Costa IG, Grosveld FG, Papantonis A. Genome Res. 2016;26(11):1478-89. https:// doi.org/101101/gr210005116.
13. Goldstein I, Baek S, Presman DM, Paakinaho V, Swinstead EE, Hager GL. Genome Res. 2017;27(3):427-39. https://doi.org/101101/gr212175116.

14. Rendeiro AF, Schmidl C, Strefford JC, Walewska R, Davis Z, Farlik M, Oscier D, Bock C. Chromatin accessibility maps of chronic lymphocytic leukaemia identify subtype-specific epigenome signatures and transcription regulatory networks. Nat Commun. 2016;7:11938.

15. Quach B, Furey TS. Bioinformatics. 2016;33(7):956-63.

16. Raj A, Shim H, Gilad Y, Pritchard JK, Stephens M. PloS ONE. 2015;10(9): 0138030.

17. Reznikoff WS. Annu Rev Genet. 2008;42:269-86.

18. Schep AN, Buenrostro JD, Denny SK, Schwartz K, Sherlock G, Greenleaf WJ. Genome Res. 2015;25(11):1757-70. https://doi.org/101101/ gr192294115.

19. He HH, Meyer CA, Chen M-W, Zang C, Liu Y, Rao PK, Fei T, Xu H, Long H, Liu XS, et al. Nat Methods. 2014;11(1):73-8.

20. Sung M-H, Guertin MJ, Baek S, Hager GL. Mol Cell. 2014;56(2):275-85.

21. Martins AL, Walavalkar NM, Anderson WD, Zang C, Guertin MJ. Nucleic Acids Res. 2018;46(2):9. https://doi.org/101093/nar/gkx1053.

22. Koohy H, Down Ta, Hubbard TJ. PLoS ONE. 2013;8(7):1-9. https://doi. org/101371/journalpone0069853.

23. Wang JR, Quach B, Furey TS. BMC Bioinforma. 2017;18(1):357.

24. In: Burnham KP, Anderson DR, editors. Model selection and multimodel inference. New York: Springer; 2004. https://doi.org/101007/b97636. http://linkspringercom/101007/b97636.

25. Ben-Gal I, Shani A, Gohr A, Grau J, Arviv S, Shmilovici A, Posch S, Grosse I. Bioinformatics. 2005;21(11):2657-66.

26. Keilwagen J, Grau J. Nucleic Acids Res. 2015;43(18):119.

27. Cuellar-Partida G, Buske FA, McLeay RC, Whitington T, Noble WS, Bailey TL. Bioinformatics. 2011;28(1):56-62.

28. Corces MR, Buenrostro JD, Wu B, Greenside PG, Chan SM, Koenig JL, Snyder MP, Pritchard JK, Kundaje A, Greenleaf WJ, et al. Nat Genet. 2016;48(10):1193-203.

29. Lazarovici A, Zhou T, Shafer A, Dantas Machado AC, Riley TR, Sandstrom R, Sabo PJ, Lu Y, Rohs R, Stamatoyannopoulos JA, Bussemaker HJ. Probing DNA shape and methylation state on a genomic scale with DNase I. Proc Natl Acad Sci U S A. 2013;110(16): 6376-81. https://doi.org/101073/pnas1216822110. arXiv:14081149.

30. Yardımcı GG, Frank CL, Crawford GE, Ohler U. Nucleic Acids Res. 2014;42(19):11865-78.

31. Sabo PJ, Humbert R, Hawrylycz M, Wallace JC, Dorschner MO, McArthur M, Stamatoyannopoulos JA. Proc Natl Acad Sci U S A. 2004;101(13):4537-42.

32. Crawford GE, Davis S, Scacheri PC, Renaud G, Halawi MJ, Erdos MR, Green R, Meltzer PS, Wolfsberg TG, Collins FS. Nat Methods. 2006;3(7): 503-9.

33. Buenrostro JD, Wu B, Litzenburger UM, Ruff D, Gonzales ML, Snyder MP, Chang HY, Greenleaf WJ. Nature. 2015;523(7561):486-90.

34. Sos BC, Fung H-L, Gao DR, Osothprarop TF, Kia A, He MM, Zhang K. Genome Biol. 2016;17(1):20.

35. Ernst J, Kellis M. Nat Methods. 2012;9(3):215-6.

36. Merad M, Sathe P, Helft J, Miller J, Mortha A. Annu Rev Immunol. 2013;31:563-604.

37. Felker P, Sere K, Lin Q, Becker C, Hristov M, Hieronymus T, Zenke M. J Immunol. 2010;185(9):5326-35. https://doi.org/104049/ jimmunol0903950.

38. Lin $Q$, Chauvistré $H$, Costa IG, Gusmao EG, Mitzka S, Hänzelmann S, Baying B, Klisch T, Moriggl R, Hennuy B, Smeets H, Hoffmann K, Benes V, Seré K, Zenke M. Nucleic Acids Res. 2015;43(20):1056. https://doi.org/ 101093/nar/gkv1056.

39. Khan A, Fornes O, Stigliani A, Gheorghe M, Castro-Mondragon JA, Van Der Lee R, Bessy A, Chèneby J, Kulkarni SR, Tan G, Baranasic D, Arenillas DJ, Sandelin A, Vandepoele K, Lenhard B, Ballester B, Wasserman WW, Parcy F, Mathelier A. Nucleic Acids Res. 2018;46(D1): 260-6. https://doi.org/101093/nar/gkx1126.

40. Grajales-Reyes GE, Iwata A, Albring J, WuX, Tussiwand R, Kc W, Kretzer NM, Briseño CG, Durai V, Bagadia P, Haldar M, Schönheit J, Rosenbauer F, Murphy TL, Murphy KM. Nat Immunol. 2015;16(7): 708-17. https://doi.org/101038/ni3197.

41. Cisse B, Caton ML, Lehner M, Maeda T, Scheu S, Locksley R, Holmberg D, Zweier C, den Hollander NS, Kant SG, Holter W, Rauch A, Zhuang Y, Reizis B. Cell. 2008;135(1):37-48. https://doi.org/101016/jcell200809016. 
42. WuX, Briseño CG, Grajales-Reyes GE, Haldar M, Iwata A, Kretzer NM, KCW, Tussiwand R, Higashi Y, Murphy TL, Murphy KM. Proc Natl Acad Sci. 2016;113(51):14775-80. https://doi.org/101073/pnas1611408114.

43. Foulk MS, Urban JM, Casella C, Gerbi SA. Genome Res. 2015;25(5): 725-35.

44. Rhee HS, Pugh BF. Cell. 2011;147(6):1408-19.

45. He Q, Johnston J, Zeitlinger J. Nat Biotechnol. 2015;33(4):395-401.

46. Stormo GD. DNA binding sites: representation and discovery. Bioinformatics. 2000;16(1):16-23. https://doi.org/101093/bioinformatics/ 16116.

47. Ji S, Watson LT, Carin L. IEEE Trans Pattern Anal Mach Intell. 2009;31 (2): 275-87.

48. Zhong S. Int J Pattern Recognit Artif Intell. 2005;19(02):165-82.

49. Rabiner LR. Proc IEEE. 1989;77(2):257-86.

50. Boyle AP, Song L, Lee B-K, London D, Keefe D, Birney E, lyer VR, Crawford GE, Furey TS. Genome Res. 2011;21(3):456-64.

51. Sherwood RI, Hashimoto T, O'donnell CW, Lewis S, Barka I AA, Van Hoff $J P$, Karun V, Jaakkola T, Gifford DK. Discovery of directional and nondirectional pioneer transcription factors by modeling dnase profile magnitude and shape. Nat Biotechnol. 2014;32(2):171-8

52. Love Ml, Huber W, Anders S. Moderated estimation of fold change and dispersion for rna-seq data with deseq2. Genome Biol. 2014;15(12):550.

53. Schmidl C, Rendeiro AF, Sheffield NC, Bock C. ChIPmentation: fast, robust, low-input ChIP-seq for histones and transcription factors. Nat Methods. 2015;12(10):963-5.

54. Lara-Astiaso D, Weiner A, Lorenzo-Vivas E, Zaretsky I, Jaitin DA, David E, Keren-Shaul H, Mildner A, Winter D, Jung S, et al. Chromatin state dynamics during blood formation. Science. 2014;345(6199):943-9.

55. Thurman RE, Rynes E, Humbert R, Vierstra J, Maurano MT, Haugen E, Sheffield NC, Stergachis AB, Wang $H$, Vernot B, et al. The accessible chromatin landscape of the human genome. Nature. 2012;489(7414):75.

56. Krueger F. Trim Galore. 2016. https://githubcom/FelixKrueger/ TrimGalore. Accessed 27 May 2017.

57. Langmead B, Salzberg SL. Fast gapped-read alignment with Bowtie 2. Nat Methods. 2012;9(4):357-9.

58. Li H, Handsaker B, Wysoker A, Fennell T, Ruan J, Homer N, Marth G, Abecasis G, Durbin R, Subgroup GPDP. The Sequence Alignment/Map format and SAMtools. Bioinformatics. 2009;25(16):2078-9.

59. Zhang Y, Liu T, Meyer CA, Eeckhoute J, Johnson DS, Bernstein BE, Nusbaum C, Myers RM, Brown M, Li W, et al. Model-based analysis of chip-seq (macs). Genome Biol. 2008;9(9):137.

60. Consortium EP, et al. An integrated encyclopedia of DNA elements in the human genome. Nature. 2012;489(7414):57-74

61. Mathelier A, Zhao X, Zhang AW, Parcy F, Worsley-Hunt R, Arenillas DJ, Buchman S, Chen C-y, Chou A, lenasescu H, et al. Jaspar 2014: an extensively expanded and updated open-access database of transcription factor binding profiles. Nucleic Acids Res. 2013;42(D1): 142-7.

62. Robasky K, Bulyk ML. UniPROBE, update 2011: expanded content and search tools in the online database of protein-binding microarray data on protein-DNA interactions. Nucleic Acids Res. 2010;39(suppl_1):124-8.

63. Matys V, Kel-Margoulis OV, Fricke E, Liebich I, Land S, Barre-Dirrie A Reuter I, Chekmenev D, Krull M, Hornischer K, et al. TRANSFAC ${ }^{\oplus}$ and its module TRANSCompel ${ }^{\otimes}$ : transcriptional gene regulation in eukaryotes. Nucleic Acids Res. 2006;34(suppl_1):108-10.

64. Korhonen J, Martinmäki P, Pizzi C, Rastas P, Ukkonen E. MOODS: fast search for position weight matrix matches in DNA sequences. Bioinformatics. 2009;25(23):3181-2.

65. Wilczynski B, Dojer N, Patelak M, Tiuryn J. Finding evolutionarily conserved cis-regulatory modules with a universal set of motifs. BMC Bioinforma. 2009;10(1):82.

66. Davis J, Goadrich M. The relationship between Precision-Recall and ROC curves. In: Proceedings of the 23 rd International Conference on Machine Learning. ACM; 2006. p. 233-40.

67. Saito T, Rehmsmeier M. The precision-recall plot is more informative than the ROC plot when evaluating binary classifiers on imbalanced datasets. PloS ONE. 2015;10(3):0118432.

68. Li Z, Schulz MH, LookT, Begemann M, Zenke M, Costa IG. HINT-ATAC. https://doi.org/105281/zenodo2540828.

69. Li Z, Schulz MH, LookT, Begemann M, Zenke M, Costa IG. Identification of Transcription Factor Binding Sites using ATAC-seq
Accession: GSE1 18221. https://wwwncbinlmnihgov/geo/query/acccgi? acc=GSE118221. Accessed 20 May 2018.

70. Snyder M. ARID3A ChIP-seq on Human K562 Produced by the Snyder Lab ENCODE Accession: ENCSRO00EFY. https://wwwencodeprojectorg/ experiments/ENCSRO0OEFY. Accessed 20 Jul 2017.

71. Struhl K. ATF1 ChIP-seq on Human K562 ENCODE Accession: ENCSRO00DNZ. https://wwwencodeprojectorg/experiments/ ENCSRO00DNZ. Accessed $20 \mathrm{Jul} 2017$.

72. Struhl K. ATF3 ChIP-seq on Human K562 ENCODE Accession: ENCSRO0ODOG. https://wwwencodeprojectorg/experiments/ ENCSRO00DOG. Accessed 20 Jul 2017.

73. Snyder M. BACH1 ChIP-seq on human K562 produced by the Snyder Lab ENCODE Accession: ENCSRO00EGD. https://wwwencodeprojectorg/ experiments/ENCSRO0OEGD. Accessed 20 Jul 2017.

74. Snyder M. ENCODE transcription factor binding sites by ChIP-sea ENCODE accession: ENCSRO0OEGD. https://wwwencodeprojectorg/ experiments/ENCSRO0OEGV. Accessed 20 Jul 2017.

75. Struhl K. CCNT2 ChIP-seq on human K562 ENCODE accession: ENCSRO00DOA. https://wwwencodeprojectorg/experiments/ ENCSRO00DOA. Accessed 20 Jul 2017.

76. Snyder M. CEBPB ChIP-seq on human K562 ENCODE accession: ENCSRO00EHE. https://wwwencodeprojectorg/experiments/ ENCSRO00EHE. Accessed 20 Jul 2017.

77. Snyder M. CTCF ChIP-seq on human K562 produced by the Snyder Lab ENCODE accession: ENCSRO00EGM. https://wwwencodeprojectorg/ experiments/ENCSRO0OEGM. Accessed 20 Jul 2017.

78. Myers R. CTCFL ChIP-seq protocol V0416101 on human K562 ENCODE accession: ENCSRO00BNK. https://wwwencodeprojectorg/experiments/ ENCSRO0OBNK. Accessed 20 Jul 2017.

79. Farnham P. E2F4 ChIP-seq on human K562 ENCODE accession: ENCSRO00EWL. https://wwwencodeprojectorg/experiments/ ENCSRO00EWL. Accessed 20 Jul 2017.

80. Farnham P. E2F6 ChIP-seq on human K562 ENCODE accession: ENCSRO0OEWJ. https://wwwencodeprojectorg/experiments/ ENCSRO0OEWJ. Accessed 20 Jul 2017.

81. Myers R. FOS ChIP-seq on human K562 EGFP-FOS ENCODE accession: ENCSRO00DKB. https://wwwencodeprojectorg/experiments/ ENCSRO0ODKB. Accessed 20 Jul 2017.

82. Myers R. GATA2 ChIP-seq on human K562 EGFP-GATA2 ENCODE accession: ENCSRO00DKA. https://wwwencodeprojectorg/experiments/ ENCSRO00DKA. Accessed 20 Jul 2017.

83. Myers R. EGR1 ChIP-seq protocol V0416101 on human K562 ENCODE accession: ENCSRO0OBNE. https://wwwencodeprojectorg/experiments/ ENCSRO0OBNE. Accessed 20 Jul 2017.

84. Myers R. JUNB ChIP-seq on human K562 EGFP-JunB ENCODE accession: ENCSRO00DJY. https://wwwencodeprojectorg/experiments/ ENCSRO00DJY. Accessed 20 Jul 2017.

85. Myers R. JUND ChIP-seq on human K562 EGFP-JunD ENCODE accession: ENCSROOODJX. https://wwwencodeprojectorg/experiments/ ENCSRO00DJX. Accessed 20 Jul 2017.

86. Myers R. ELF1 ChIP-seq protocol V0416102 on human K562 ENCODE accession: ENCSRO00BMD. https://wwwencodeprojectorg/experiments/ ENCSRO00BMD. Accessed 20 Jul 2017.

87. Snyder M. ELK1 ChIP-seq on human K562 produced by the Snyder Lab ENCODE accession: ENCSRO00EFU. https://wwwencodeprojectorg/ experiments/ENCSROOOEFU. Accessed $20 \mathrm{Jul} 2017$.

88. Myers R. ETS1 ChIP-seq protocol V0416101 on human K562 ENCODE accession: ENCSRO0OBKQ. https://wwwencodeprojectorg/experiments/ ENCSRO0OBKQ. Accessed 20 Jul 2017.

89. Weissman S. FOS ChIP-seq on human K562 ENCODE accession: ENCSROOOFAI. https://wwwencodeprojectorg/experiments/ ENCSRO0OFAI. Accessed $20 \mathrm{Jul} 2017$.

90. Myers R. FOSL1 ChIP-seq protocol V0416101 on human K562 ENCODE accession: ENCSRO0OBMV. https://wwwencodeprojectorg/experiments/ ENCSROOOBMV. Accessed 20 Jul 2017

91. Myers R. GABPA ChIP-seq protocol V0416101 on human K562 ENCODE accession: ENCSRO0OBLO. https://wwwencodeprojectorg/experiments/ ENCSRO0OBLO. Accessed 20 Jul 2017.

92. Farnham P. GATA1 ChIP-seq on human K562 ENCODE accession: ENCSRO00EWM. https://wwwencodeprojectorg/experiments/ ENCSRO00EWM. Accessed 20 Jul 2017. 
93. Farnham P. GATA2 ChIP-seq on human K562 ENCODE accession: ENCSRO00EWG. https://wwwencodeprojectorg/experiments/ ENCSRO00EWG. Accessed $20 \mathrm{Jul} 2017$.

94. Snyder M. IRF1 ChIP-seq on human K562 treated with IFNa6h produced by the Snyder Lab ENCODE accession: ENCSR000EGL. https:// wwwencodeprojectorg/experiments/ENCSR000EGL. Accessed $20 \mathrm{Jul}$ 2017.

95. Weissman S. JUN ChIP-seq on human K562 ENCODE accession: ENCSRO0OFAH. https://wwwencodeprojectorg/experiments/ ENCSRO00FAH. Accessed 20 Jul 2017.

96. Snyder M. JUND ChIP-seq on human K562 produced by the Snyder Lab ENCODE accession: ENCSRO00EGN. https://wwwencodeprojectorg/ experiments/ENCSRO00EGN. Accessed 20 Jul 2017.

97. Snyder M. MAFF ChIP-seq on human K562 produced by the Snyder Lab ENCODE accession: ENCSRO00EGI. https://wwwen codeprojectorg/experiments/ENCSRO00EGI. Accessed 20 Jul 2017.

98. Snyder M. MAFK ChIP-seq on human K562 ENCODE accession: ENCSRO00EGX. https://wwwencodeprojectorg/experiments/ ENCSRO00EGX. Accessed 20 Jul 2017.

99. Snyder M. MAX ChIP-seq on Human K562 produced by the Snyder Lab ENCODE accession: ENCSRO00EFV. https://wwwencodeprojectorg/ experiments/ENCSRO00EFV. Accessed 20 Jul 2017.

100. Myers R. MEF2A ChIP-seq protocol V0416101 on human K562 ENCODE accession: ENCSRO00BNV. https://wwwencodeprojectorg/experiments/ ENCSRO00BNV. Accessed 20 Jul 2017.

101. Weissman S. MYC ChIP-seq on human K562 ENCODE accession: ENCSRO0OFAG. https://wwwencodeprojectorg/experiments/ ENCSRO00FAG. Accessed 20 Jul 2017

102. Weissman S. NFE2 ChIP-seq on human K562 ENCODE accession: ENCSR000FAF. https://wwwencodeprojectorg/experiments/ ENCSRO00FAF. Accessed 20 Jul 2017.

103. Snyder M. NFYA ChIP-seq on human K562 ENCODE accession: ENCSRO00EGR. https://wwwencodeprojectorg/experiments/ ENCSRO00EGR. Accessed $20 \mathrm{Jul} 2017$.

104. Snyder M. NFYB ChIP-seq on human K562 ENCODE accession: ENCSR000EGQ. https://wwwencodeprojectorg/experiments/ ENCSRO00EGQ. Accessed 20 Jul 2017.

105. Myers R. NR2F2 ChIP-seq protocol V0422111 on human K562 ENCODE accession: ENCSRO00BRS. https://wwwencodeprojectorg/experiments/ ENCSRO0OBRS. Accessed 20 Jul 2017

106. Snyder M. NRF1 ChIP-seq on human K562 ENCODE accession: ENCSRO00EHH. https://wwwencodeprojectorg/experiments/ ENCSRO00EHH. Accessed 20 Jul 2017.

107. Myers R. SPI1 ChIP-seq protocol PCR1x on human K562 ENCODE accession: ENCSRO00BGW. https://wwwencodeprojectorg/experiments/ ENCSRO00BGW. Accessed 20 Jul 2017.

108. Weissman S. RAD21 ChIP-seq on human K562 ENCODE accession: ENCSRO0OFAD. https://wwwencodeprojectorg/experiments/ ENCSRO00FAD. Accessed 20 Jul 2017.

109. Myers R. REST ChIP-seq protocol V0416102 on human K562 ENCODE accession: ENCSR000BMW. https://wwwencodeprojectorg/ experiments/ENCSRO00BMW. Accessed 20 Jul 2017.

110. Snyder M. RFX5 ChIP-seq on human K562 ENCODE accession: ENCSR000EGO. https://wwwencodeprojectorg/experiments/ ENCSRO00EGO. Accessed 20 Jul 2017.

111. Myers R. SIX5 ChIP-seq protocol PCR1x on human K562 ENCODE accession: ENCSR000BGX. https://wwwencodeprojectorg/experiments/ ENCSRO00BGX. Accessed 20 Jul 2017.

112. Snyder M. SMC3 ChIP-seq on human K562 ENCODE accession: ENCSRO00EGW. https://wwwencodeprojectorg/experiments/ ENCSRO00EGW. Accessed 20 Jul 2017.

113. Myers R. SP1 ChIP-seq protocol PCR1x on human K562 ENCODE accession: ENCSRO00BKO. https://wwwencodeprojectorg/experiments/ ENCSRO00BKO. Accessed 20 Jul 2017.

114. Myers R. SP2 ChIP-seq protocol V0416102 on human K562 ENCODE accession: ENCSRO00BNL. https://wwwencodeprojectorg/experiments/ ENCSRO00BNL. Accessed 20 Jul 2017.

115. Myers R. SRF ChIP-seq protocol V0416101 on human K562 ENCODE accession: ENCSR000BLK. https://wwwencodeprojectorg/experiments/ ENCSRO00BLK. Accessed 20 Jul 2017.
116. Weissman S. STAT1 ChIP-seq on human K562 treated with IFNa for 6 hours ENCODE accession: ENCSRO00FAU. https:// wwwencodeprojectorg/experiments/ENCSRO00FAU. Accessed $20 \mathrm{Jul}$ 2017.

117. Weissman S. STAT2 ChIP-seq on human K562 treated with IFNa for 6 hours ENCODE accession: ENCSRO0OFBC. https:// wwwencodeprojectorg/experiments/ENCSRO00FBC. Accessed 20 Jul 2017.

118. Myers R. STAT5A ChIP-seq protocol V0422111 on human K562 ENCODE accession: ENCSR000BRR. https://wwwencodeprojectorg/experiments/ ENCSRO00BRR. Accessed $20 \mathrm{Jul} 2017$

119. Snyder M. TAL1 ChIP-seq on human K562 ENCODE accession: ENCSRO00EHB. https://wwwencodeprojectorg/experiments/ ENCSRO00EHB. Accessed 20 Jul 2017.

120. Snyder M. TBP ChIP-seq on human K562 ENCODE accession: ENCSRO00EHA. https://wwwencodeprojectorg/experiments/ ENCSRO00EHA. Accessed 20 Jul 2017

121. Myers R. THAP1 ChIP-seq protocol V0416101 on human K562 ENCODE accession: ENCSR000BNN. https://wwwencodeprojectorg/experiments/ ENCSROOOBNN. Accessed 20 Jul 2017.

122. Farnham P. NR2C2 ChIP-seq on human K562 ENCODE accession: ENCSR000EWH. https://wwwencodeprojectorg/experiments/ ENCSRO00EWH. Accessed 20 Jul 2017.

123. Myers R. USF1 ChIP-seq protocol V0416101 on human K562 ENCODE accession: ENCSRO00BKT. https://wwwencodeprojectorg/experiments/ ENCSRO00BKT. Accessed 20 Jul 2017

124. Snyder M. USF2 ChIP-seq on human K562 ENCODE accession: ENCSRO00EHG. https://wwwencodeprojectorg/experiments/ ENCSRO00EHG. Accessed 20 Jul 2017

125. Farnham P. YY1 ChIP-seq on human K562 ENCODE accession: ENCSR000EWF. https://wwwencodeprojectorg/experiments/ ENCSRO00EWF. Accessed 20 Jul 2017.

126. Myers R. ZBTB33 ChIP-seq protocol PCR1x on human K562 ENCODE accession: ENCSR000BKF. https://wwwencodeprojectorg/experiments/ ENCSR000BKF. Accessed 20 Jul 2017.

127. Myers R. ZBTB7A ChIP-seq protocol V0416101 on human K562 ENCODE accession: ENCSRO00BME. https://wwwencodeprojectorg/experiments/ ENCSRO00BME. Accessed 20 Jul 2017.

128. Snyder M. ZNF143 ChIP-seq on human K562 ENCODE accession: ENCSR000EGP. https://wwwencodeprojectorg/experiments/ ENCSRO00EGP. Accessed 20 Jul 2017.

129. Farnham P. ZNF263 ChIP-seq on human K562 ENCODE accession: ENCSRO00EWN. https://wwwencodeprojectorg/experiments/ ENCSROOOEWN. Accessed 20 Jul 2017.

130. Myers R. ATF3 ChIP-seq protocol PCR1x on human GM12878 ENCODE accession: ENCSRO0OBJY. https://wwwencodeprojectorg/experiments/ ENCSRO0OBJY. Accessed 20 Jul 2017.

131. Snyder M. BHLHE40 ChIP-seq on human GM12878 ENCODE accession: ENCSRO00DZJ. https://wwwencodeprojectorg/experiments/ ENCSRO00DZJ. Accessed $20 \mathrm{Jul} 2017$

132. Myers R. CEBPB ChIP-seq Protocol V0422111 on human GM12878 ENCODE Accession: ENCSRO00BRX. https://wwwencodeprojectorg/ experiments/ENCSRO00BRX. Accessed 20 Jul 2017.

133. Snyder M. CTCF ChIP-seq on human GM12878 ENCODE accession: ENCSR000DZN. https://wwwencodeprojectorg/experiments/ ENCSRO00DZN. Accessed 20 Jul 2017.

134. Snyder M. E2F4 ChIP-seq on Human GM12878 produced by the Snyder Lab ENCODE accession: ENCSR000DYY. https://wwwencodeprojectorg/ experiments/ENCSRO00DYY. Accessed 20 Jul 2017.

135. Myers R. EGR1 ChIP-seq protocol PCR2x on human GM12878 ENCODE accession: ENCSRO00BRG. https://wwwencodeprojectorg/experiments/ ENCSRO00BRG. Accessed 20 Jul 2017.

136. Myers R. ELF1 ChIP-seq protocol V0416101 on human GM12878 ENCODE accession: ENCSRO00BMB. https://wwwencodeprojectorg/ experiments/ENCSROOOBMB. Accessed 20 Jul 2017.

137. Snyder M. ELK1 ChIP-seq on human GM12878 produced by the Snyder Lab ENCODE accession: ENCSR000DZB. https://wwwencodeprojectorg/ experiments/ENCSRO00DZB. Accessed 20 Jul 2017.

138. Myers R. ETS1 ChIP-seq protocol PCR1x on human GM12878 ENCODE accession: ENCSRO00BKA. https://wwwencodeprojectorg/experiments/ ENCSRO00BKA. Accessed 20 Jul 2017. 
139. Weissman S. FOS ChIP-seq on human GM12878 ENCODE accession: ENCSR000EYZ. https://wwwencodeprojectorg/experiments/ ENCSRO00EYZ. Accessed 20 Jul 2017.

140. Weissman S. JUND ChIP-seq on human GM12878 ENCODE accession: ENCSR000EYV. https://wwwencodeprojectorg/experiments/ ENCSRO00EYV. Accessed 20 Jul 2017.

141. Snyder M. MAX ChIP-seq on human GM12878 produced by the Snyder Lab ENCODE accession: ENCSRO00DZF. https://wwwencodeprojectorg/ experiments/ENCSRO00DZF. Accessed 20 Jul 2017

142. Myers R. MEF2A ChIP-seq protocol PCR1x on human GM12878 ENCODE accession: ENCSRO00BKB. https://wwwencodeprojectorg/experiments/ ENCSRO00BKB. Accessed 20 Jul 2017.

143. Iyer V. MYC ChIP-seq on human GM12878 ENCODE accession: ENCSR000DKU. https://wwwencodeprojectorg/experiments/ ENCSR000DKU. Accessed 20 Jul 2017.

144. Snyder M. NFE2 ChIP-seq on human GM12878 ENCODE accession: ENCSR000DZY. https://wwwencodeprojectorg/experiments/ ENCSRO00DZY. Accessed 20 Jul 2017.

145. Struhl K. NFYA ChIP-seq on human GM12878 ENCODE accession: ENCSR000DNN. https://wwwencodeprojectorg/experiments/ ENCSRO00DNN. Accessed 20 Jul 2017

146. Struhl K. NFYB ChIP-seq on human GM12878 ENCODE accession: ENCSRO00DNM. https://wwwencodeprojectorg/experiments/ ENCSRO00DNM. Accessed 20 Jul 2017

147. Snyder M. NRF1 ChIP-seq on human GM12878 ENCODE accession: ENCSR000DZO. https://wwwencodeprojectorg/experiments/ ENCSR000DZO. Accessed 20 Jul 2017.

148. Myers R. REST ChIP-seq Protocol PCR1x on human GM12878 ENCODE accession: ENCSRO0OBQS. https://wwwencodeprojectorg/experiments/ ENCSRO00BQS. Accessed 20 Jul 2017.

149. Myers R. SPI1 ChIP-seq Protocol PCR1x on human GM12878 ENCODE accession: ENCSRO00BGQ. https://wwwencodeprojectorg/experiments/ ENCSRO00BGQ. Accessed 20 Jul 2017.

150. Snyder M. RAD21 ChIP-seq on human GM12878 ENCODE accession: ENCSRO00EAC. https://wwwencodeprojectorg/experiments/ ENCSRO00EAC. Accessed 20 Jul 2017.

151. Snyder M. RFX5 ChIP-seq on human GM12878 produced by the Snyder Lab ENCODE accession: ENCSRO00DZW. https://wwwencodeprojectorg/ experiments/ENCSRO00DZW. Accessed 20 Jul 2017.

152. Myers R. SIX5 ChIP-seq protocol PCR1X on human GM12878 ENCODE accession: ENCSRO00BJE. https://wwwencodeprojectorg/experiments/ ENCSRO00BJE. Accessed 20 Jul 2017.

153. Snyder M. SMC3 ChIP-seq on Human GM12878 produced by the Snyder Lab ENCODE accession: ENCSR000DZP. https://wwwencodeprojectorg/ experiments/ENCSRO00DZP. Accessed 20 Jul 2017.

154. Myers R. SP1 ChIP-seq protocol PCR1x on human GM12878 ENCODE accession: ENCSRO00BHK. https://wwwencodeprojectorg/experiments/ ENCSRO0OBHK. Accessed 20 Jul 2017.

155. Myers R. SRF ChIP-seq protocol PCR2x on human GM12878 ENCODE accession: ENCSRO00BGE. https://wwwencodeprojectorg/experiments/ ENCSRO00BGE. Accessed 20 Jul 2017.

156. Snyder M. STAT1 ChIP-seq on human GM12878 ENCODE accession: ENCSR000DZM. https://wwwencodeprojectorg/experiments/ ENCSRO00DZM. Accessed 20 Jul 2017

157. Myers R. STAT5A ChIP-seq protocol V0422111 on human GM12878 ENCODE accession: ENCSRO00BQZ. https://wwwencodeprojectorg/ experiments/ENCSRO00BQZ. Accessed 20 Jul 2017.

158. Farnham P. NR2C2 ChIP-seq on human GM12878 ENCODE accession: ENCSR000EUL. https://wwwencodeprojectorg/experiments/ ENCSRO00EUL. Accessed 20 Jul 2017.

159. Snyder M. USF2 ChIP-seq on human GM12878 produced by the Snyder Lab ENCODE accession: ENCSRO00DZU. https://wwwencodeprojectorg/ experiments/ENCSRO00DZU. Accessed 20 Jul 2017.

160. Farnham P. YY1 ChIP-seq on human GM12878 ENCODE accession: ENCSRO00EUM. https://wwwencodeprojectorg/experiments/ ENCSROOOEUM. Accessed 20 Jul 2017

161. Myers R. ZBTB33 ChIP-seq protocol PCR1x on human GM12878 ENCODE accession: ENCSRO00BHC. https://wwwencodeprojectorg/experiments/ ENCSRO00BHC. Accessed 20 Jul 2017.

162. Snyder M. ZNF143 ChIP-seq on human GM12878 ENCODE accession: ENCSR000DZL. https://wwwencodeprojectorg/experiments/ ENCSRO00DZL. Accessed 20 Jul 2017
163. Myers R. ATF3 ChIP-seq protocol V0416102 on human H1-hESC ENCODE accession: ENCSRO00BKC. https://wwwencodeprojectorg/experiments/ ENCSRO00BKC. Accessed 20 Jul 2017.

164. Snyder M. BACH1 ChIP-seq on human H1-hESC produced by the Snyder Lab ENCODE accession: ENCSRO00EBQ. https://wwwencodeprojectorg/ experiments/ENCSRO00EBQ. Accessed 20 Jul 2017.

165. Snyder M. BRCA1 ChIP-seq on human H1-hESC produced by the Snyder Lab ENCODE accession: ENCSRO00EBX. https://wwwencodeprojectorg/ experiments/ENCSRO00EBX. Accessed 20 Jul 2017.

166. Snyder M. CEBPB ChIP-seq on human H1-hESC produced by the Snyder Lab ENCODE accession: ENCSRO00EBV. https://wwwencodeprojectorg/ experiments/ENCSRO00EBV. Accessed 20 Jul 2017.

167. Myers R. CTCF ChIP-seq protocol V0416102 on human H1-hESC ENCODE accession: ENCSRO0OBNH. https://wwwencodeprojectorg/experiments/ ENCSROOOBNH. Accessed $20 \mathrm{Jul} 2017$.

168. Myers R. EGR1 ChIP-seq protocol V0416102 on human H1-hESC ENCODE accession: ENCSRO00BJA. https://wwwencodeprojectorg/experiments/ ENCSRO00BJA. Accessed 20 Jul 2017.

169. Myers R. FOSL1 ChIP-seq protocol V0416102 on human H1-hESC ENCODE accession: ENCSRO00BNS. https://wwwencodeprojectorg/ experiments/ENCSRO00BNS. Accessed 20 Jul 2017.

170. Myers R. GABPA ChIP-seq protocol PCR1x on human H1-hESC ENCODE accession: ENCSRO00BIW. https://wwwencodeprojectorg/experiments/ ENCSRO00BIW. Accessed 20 Jul 2017.

171. Snyder M. JUN ChIP-seq on human H1-hESC ENCODE accession: ENCSR000ECA. https://wwwencodeprojectorg/experiments/ ENCSRO00ECA. Accessed 20 Jul 2017.

172. Snyder M. JUND ChIP-seq on human H1-hESC ENCODE accession: ENCSR000EBZ. https://wwwencodeprojectorg/experiments/ ENCSRO00EBZ. Accessed 20 Jul 2017.

173. Snyder M. MAFK ChIP-seq on human H1-hESC Produced by the Snyder Lab ENCODE accession: ENCSR000EBS. https://wwwencodeprojectorg/ experiments/ENCSRO00EBS. Accessed 20 Jul 2017.

174. Farnham P. MAX ChIP-seq on human H1-hESC ENCODE accession: ENCSR000EUP. https://wwwencodeprojectorg/experiments/ ENCSRO00EUP. Accessed 20 Jul 2017.

175. Snyder M. MYC ChIP-seq on human H1-hESC produced by the Snyder Lab ENCODE accession: ENCSRO0OEBY. https://wwwencodeprojectorg/ experiments/ENCSRO00EBY. Accessed 20 Jul 2017.

176. Snyder M. NRF1 ChIP-seq on human H1-hESC ENCODE accession: ENCSR000ECC. https://wwwencodeprojectorg/experiments/ ENCSRO00ECC. Accessed 20 Jul 2017.

177. Myers R. EP300 ChIP-seq protocol V0416102 on human H1-hESC ENCODE accession: ENCSRO00BKK. https://wwwencodeprojectorg/ experiments/ENCSRO00BKK. Accessed 20 Jul 2017.

178. Myers R. POU5F1 ChIP-seq protocol V0416102 on human H1-hESC ENCODE accession: ENCSRO00BMU. https://wwwencodeprojectorg/ experiments/ENCSRO00BMU. Accessed 20 Jul 2017.

179. Snyder M. RAD21 ChIP-seq on human H1-hESC ENCODE accession: ENCSRO00ECE. https://wwwencodeprojectorg/experiments/ ENCSRO00ECE. Accessed 20 Jul 2017.

180. Myers R. REST ChIP-seq protocol V0416102 on human H1-hESC ENCODE accession: ENCSRO00BHM. https://wwwencodeprojectorg/experiments/ ENCSRO00BHM. Accessed 20 Jul 2017

181. Snyder M. RFX5 ChIP-seq on human H1-hESC ENCODE accession: ENCSRO00ECF. https://wwwencodeprojectorg/experiments/ ENCSRO00ECF. Accessed $20 \mathrm{Jul} 2017$.

182. Myers R. RXRA ChIP-seq protocol V0416102 on human H1-hESC ENCODE accession: ENCSR000BJW. https://wwwencodeprojectorg/ experiments/ENCSRO00BJW. Accessed 20 Jul 2017.

183. Myers R. SIX5 ChIP-seq protocol PCR1x on human H1-hESC ENCODE accession: ENCSRO00BIQ. https://wwwencodeprojectorg/experiments/ ENCSRO00BIQ. Accessed 20 Jul 2017.

184. Myers R. SP1 ChIP-seq protocol PCR1x on human H1-hESC ENCODE accession: ENCSRO00BIR. https://wwwencodeprojectorg/experiments/ ENCSRO00BIR. Accessed 20 Jul 2017.

185. Myers R. SP2 ChIP-seq protocol V0422111 on human H1-hESC ENCODE accession: ENCSRO0OBQG. https://wwwencodeprojectorg/experiments/ ENCSRO00BQG. Accessed 20 Jul 2017

186. Myers R. SP4 ChIP-seq protocol V0422111 on human H1-hESC ENCODE accession: ENCSRO00BQV. https://wwwencodeprojectorg/experiments/ ENCSRO00BQV. Accessed 20 Jul 2017. 
187. Myers R. SRF ChIP-seq Protocol PCR1x on human H1-hESC ENCODE accession: ENCSRO0OBIV. https://wwwencodeprojectorg/experiments/ ENCSRO0OBIV. Accessed 20 Jul 2017.

188. Snyder M. TBP ChIP-seq on human H1-hESC ENCODE accession: ENCSRO0OECB. https://wwwencodeprojectorg/experiments/ ENCSRO00ECB. Accessed 20 Jul 2017.

189. Myers R. TCF12 ChIP-seq protocol PCR1x on human H1-hESC ENCODE accession: ENCSRO00BIT. https://wwwencodeprojectorg/experiments/ ENCSRO0OBIT. Accessed 20 Jul 2017.

190. Myers R. USF1 ChIP-seq Protocol PCR1x on human H1-hESC ENCODE accession: ENCSRO0OBIU. https://wwwencodeprojectorg/experiments/ ENCSRO0OBIU. Accessed 20 Jul 2017.

191. Snyder M. USF2 ChIP-seq on human H1-hESC ENCODE accession: ENCSROOOECD. https://wwwencodeprojectorg/experiments/ ENCSRO00ECD. Accessed 20 Jul 2017

192. Myers R. YY1 ChIP-seq protocol V0416102 on human H1-hESC ENCODE accession: ENCSRO0OBKD. https://wwwencodeprojectorg/experiments/ ENCSRO0OBKD. Accessed 20 Jul 2017.

193. Snyder M. ZNF143 ChIP-seq on human H1-hESC produced by the Snyder Lab ENCODE accession: ENCSRO00EBW. https://www. encodeproject.org/experiments/ENCSRO00EBW. Accessed 20 Jul 2017.

Ready to submit your research? Choose BMC and benefit from:

- fast, convenient online submission

- thorough peer review by experienced researchers in your field

- rapid publication on acceptance

- support for research data, including large and complex data types

- gold Open Access which fosters wider collaboration and increased citations

- maximum visibility for your research: over $100 \mathrm{M}$ website views per year

At BMC, research is always in progress.

Learn more biomedcentral.com/submissions 